WILCELLY MACHADO DA SILVA

PERFIL DE MEDIADORES INFLAMATÓRIOS NO PROCESSO ATEROSCLERÓTICO CAROTÍDEO

BRASÍLIA-DF 
UNIVERSIDADE DE BRASÍLIA

FACULDADE DE MEDICINA

WILCELLY MACHADO DA SILVA

\title{
PERFIL DE MEDIADORES INFLAMATÓRIOS NO PROCESSO
}

ATEROSCLERÓTICO CAROTÍDEO

\begin{abstract}
Dissertação apresentada como requisito para a obtenção do Título de Mestre em Ciências da Saúde pelo Programa de Pós-Graduação em Ciências da Saúde da Universidade de Brasília.
\end{abstract}

Orientador: Professor Doutor Otávio de Tolêdo Nóbrega

BRASÍLIA-DF 
Wilcelly Machado da Silva

\section{PERFIL DE MEDIADORES INFLAMATÓRIOS NO PROCESSO ATEROSCLERÓTICO CAROTÍDEO}

Dissertação apresentada como requisito para a obtenção do Título de Mestre em Ciências da Saúde pelo Programa de Pós-Graduação em Ciências da Saúde da Universidade de Brasília.

BANCA EXAMINADORA

Prof. Dr. Otávio de Tolêdo Nóbrega

Prof. Dr. Gustavo Adolfo Argañaraz

Prof. Dr. Diêgo Madureira de Oliveira

Prof. Dr. Clayton Franco Moraes 



\section{RESUMO}

Introdução: Aterosclerose é um processo inflamatório crônico iniciado no interior da parede arterial em resposta a lipoproteínas oxidadas. $O$ aumento da espessura médio-intimal carotídea (EMIc) contribui como fator de risco para doença cerebrovasculares. Objetivo: Investigar a relação de parâmetros clínicos, bioquímicos e inflamatórios com medidas de EMlc, controlando as associações para fatores de risco clássicos. Métodos: Levantamento transversal de pacientes com 60 anos de idade ou mais quanto ao uso de medicamentos para doenças crônicas, perfil bioquímico e antropométrico, eventos circulatórios anteriores, histórico familiar de doenças do aparelho circulatório e principais aspectos do estilo de vida. Realizadas medições da parede da artéria carótida direita e esquerda. Foram mensuradas concentrações de mediadores inflamatórios por citometria de fluxo. Resultados: Análise de correlação revelou associação dos níveis logaritmicamente transformados das citocinas IL16, IL6, IL8, IL10 e TNFŬ com as medidas absolutas de EMI da carótida esquerda. Outros mediadores inflamatórios avaliados (IFNo, IL2, IL4, IL12p70 e IL17a) não mostraram associação com quaisquer das medidas de EMIc. Comparação dos níveis circulatórios logaritmicamente transformados das citocinas entre os indivíduos agrupados conforme tercis de espessura médio-intimal da carótida esquerda confirmou correlação positiva com os níveis de TNFŬ e IL16, com ajustamento necessário para HDL-c. Em regressão logística multivariada, $\log _{10}$ TNFŬ foi a variável mais preditiva para explicar a variação encontrara nos valores de EMIc $\left(R^{2}=0.209\right)$, que acrescida dos $\log _{10} \mathrm{l} L 16$ e $\log _{10} \mathrm{l} 66$, nesta ordem, responderam por $29,5 \%$ da variância nas medidas do EMlc. Conclusão: Nossos resultados sugerem que níveis circulantes de IL16, IL6, IL8, IL10 e TNF apresentam correlação com a EMI da carótida esquerda em indivíduos idosos.

Palavras-chave: aterosclerose, espessura médio-intimal, artéria carótida, citocinas. 


\begin{abstract}
Background: Atherosclerosis is a chronic inflammatory process within the arterial wall initiated in response to endogenously modified structures, particularly oxidized lipoproteins that stimulate both innate and adaptive immune responses. The increasing of carotid intima-media thickness (cIMT) has been a strong predictor of future vascular events. Objective: to investigate the relationship of a profile of bloodcarried inflammatory mediators with measures of cIMT in elderly subjects, taking traditional risk factors into account. Methods: Clinical inspection for present and past chronic conditions and events as well as biochemical and anthropometric measurements were performed for patients aged 60 years or older in ambulatory care. Scores of cIMT were obtained bilaterally in the distal common carotid artery wall. Serum concentrations of ten different cytokines were assessed by bead-based, multiplexed flow cytometry immunoassays. Results: Correlation analysis demonstrated a significant level of association between log-transformed levels of the cytokines IL16, IL6, IL8, IL10 and TNFŬ $(P<0.05)$ with raw IMT scores of the left carotid. The other immune mediators investigated (IFN), IL2, IL4, IL12p70 and IL17a) showed not to associate with either cIMT scores. Accordingly, comparison of the log-transformed circulating levels of the cytokines across the individuals grouped in increasing tertiles of intima-media thickness of the left carotid artery confirmed a positive correlation with levels of TNFǓ and IL16, with adjustment necessary to HDLc. Stepwise multivariate regression showed that $\log _{10}$ TNFŬ was the first most predictive variable in the model $\left(R^{2}=0.209\right)$, which added to $\log _{10} \mathrm{IL} 16$ and $\log _{10} \mathrm{IL} 6$ in this order accounted for $29.5 \%$ of the variance in scores of intima-media thickness.
\end{abstract}

Conclusion: Our results suggest that the logarithmically transformed levels of IL16, IL6, IL8, IL10 and TNF independently correlated with the score of the left cIMT.

Keywords: atherosclerosis, intima-media thickness, carotid artery, cytokines. 


\section{LISTA DE FIGURAS}

Figura 1 ñ Comparação entre os níveis circulantes logaritmicamente transformados de TNFŬ (A) e IL16 (B) de indivíduos agrupados em tercis de espessura médio-intimal da artéria carotídea esquerda. 


\section{LISTA DE TABELAS}

Tabela 1 ñ Características clínicas, antropométricas, e metabólicas da amostra.

Tabela 2 ñ Análise de correlação dos valores brutos séricos das citocinas e das medidas absolutas de EMlc com características categóricas clínicas e de saúde na amostra à admissão.

Tabela 3 ñ Análise de correlação dos níveis séricos logaritmicamente transformados das citocinas e das medidas absolutas de EMlc com características clínicas e bioquímicas de natureza contínua na amostra à admissão.

Tabela 4 ñ Análises de correlação entre os níveis séricos logaritmicamente transformados de citocinas e medidas absolutas de EMlc à admissão. 


\section{LISTA DE ABREVIATURAS E SIGLAS}

ACC ï Artéria carótida comum;

AR ï Artrite reumatóide;

AVE ï Acidente vascular encefálico;

CA ï Circunferência abdominal;

CT ï Colesterol total;

DAC ï Doença arterial coronariana;

DM2 ï Diabetes mellitus tipo 2;

EMI ï Espessura médio-intimal;

EMIc ï Espessura médio-intimal carotídea;

HAS ï Hipertensão arterial sistêmica;

HbA1c ï Hemoglobina glicada A1c;

HDL-c ï Lipoproteína de alta densidade;

HOMA ï Modelo de avaliação homeostático;

IAM ï Infarto agudo do miocárdio;

ICAM-1 ï Molécula de adesão intercelular-1;

IFNo Ï Interferon gama;

IL- Interleucina;

IMC ï Índice de massa corporal;

LDL-c ï Lipoproteína de baixa densidade;

Log ï Dado logaritmicamente transformado

MCP-1 ï Proteína quimiotáctica de monócitos-1; 
PAD ï Pressão arterial diastólica;

PAS ï Pressão arterial sistólica;

PCR ï Proteína C reativa;

TNFǓï Fator de necrose tumoral alfa;

TSH ï Hormônio de estimulação tiroidiana

UnB ï Universidade de Brasília

UCB Ï Universidade Católica de Brasília

VLDL-c ï Lipoproteína de muita baixa densidade;

VSCM ï Células musculares lisas vasculares. 


\section{SUMÁRIO}

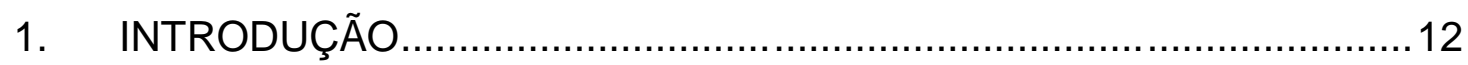

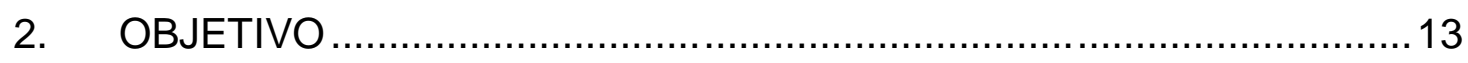

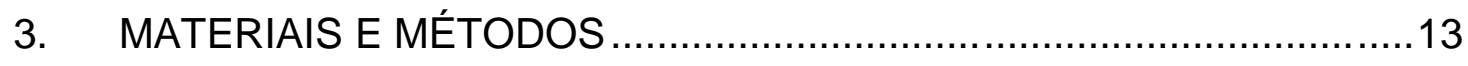

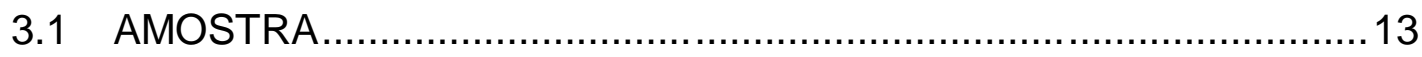

3.2 AVALIAÇÃO CLÍNICA E BIOQUÍMICA ………..............................14

3.3 MEDIÇÕES DA CAMADA MÉDIO-INTIMAL CAROTÍDEA .................15

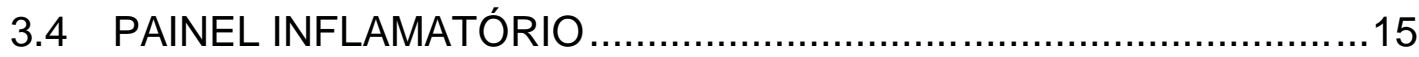

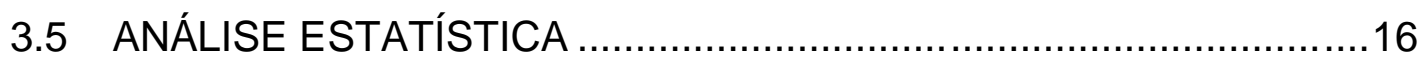

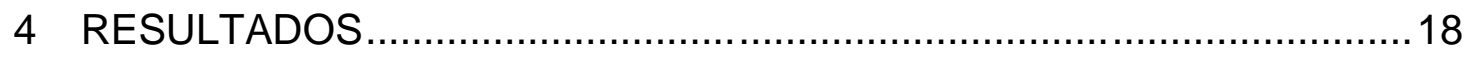

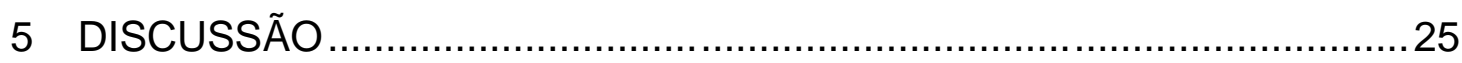

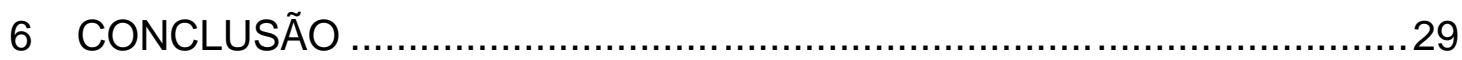

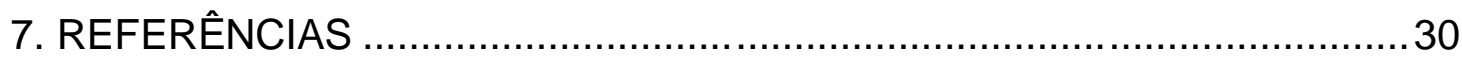

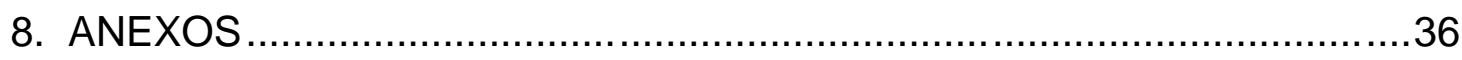

I. COMPROVANTE DE APROVAÇÃO EM COMITÊ DE ÉTICA ..................36

II. TERMO DE CONSENTIMENTO LIVRE E ESCLARECIDO .....................37

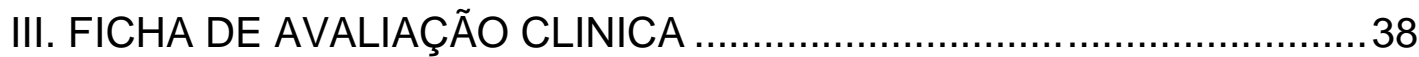

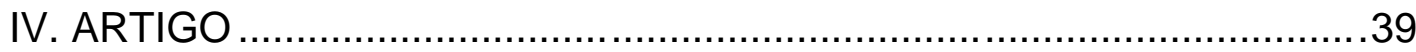




\section{INTRODUÇÃO}

A aterosclerose resulta de um processo inflamatório crônico no interior da parede arterial, e que tem início principalmente em resposta às estruturas endógenas modificadas, particularmente às lipoproteínas oxidadas. É amplamente reconhecido que a estimulação por mediadores circulantes das respostas imunológicas inata e adaptativa levam a uma maior alteração da parede vascular, promovendo a progressão da doença e complicações ${ }^{1 ; 2}$, com evidência de que o desequilíbrio entre os fatores pró- e anti-inflamatórios desempenham um papel crítico na disfunção endotelial vascular precoce $e^{3 ; 4}$.

A aterosclerose afeta artérias grandes e médias, de natureza elástica e muscular. A grande preocupação que envolve o seu surgimento e progressão consiste na possibilidade de ocorrer trombose ou estenose grave nas artérias, levando a eventos adversos que podem ameaçar a vida, como o infarto agudo do miocárdio, o acidente vascular encefálico, danos isquêmicos para os rins e intestinos, além de outras manifestações clínicas de desordens vasculares ${ }^{5}$. Portanto, evitar a morte e a morbidade por causas circulatórias justifica o grande interesse na identificação de pacientes de alto risco, mesmo em condição assintomática ${ }^{6}$.

A este respeito, técnicas de imagem constituem uma ferramenta não invasiva, segura e acessível que fornecem informações valiosas sobre leitos circulatórios periféricos, tais como as artérias carótidas, em que a identificação de aterosclerose subclínica pode melhorar a estratificação de risco para o desenvolvimento de eventos cerebrovasculares ${ }^{7 ; 8}$. Para tanto, tomamos como evidência o fato de que 0 aumento na espessura da camada médio-intimal (EMI), da ordem de $0,1 \mathrm{~mm}$, pode por si só oferecer uma contribuição relevante para o início do acidente vascular 
encefálico (AVE) (risco relativo $=1,17)^{9}$. A literatura atual, por vezes, aponta as características morfológicas da carótida como fortes preditores de futuros eventos vasculares $^{9 ; 10 ; 11 ; 12}$.

\section{OBJETIVO}

\subsection{GERAL}

Investigar a relação de um painel de marcadores inflamatórios circulantes com características clínicas e bioquímicas tradicionais de relevância para eventos vasculares, bem como com as avaliações de imagem das artérias carótidas.

\subsection{ESPECÍFICO}

A avaliação da associação entre as variáveis supracitadas permitirá as seguintes análises e desfechos clínicos:

avaliar o espessamento médio-intimal, testando a associação deste fator de risco para eventos vasculares com os níveis circulantes de citocinas eleitas para estudo, e corrigindo as análises para as demais características clínicas e bioquímico-metabólicas da amostra.

\section{MATERIAIS E MÉTODOS}

\subsection{AMOSTRA}

Pacientes consecutivos não institucionalizados, com 60 anos de idade ou mais, sem sintomas de aterosclerose severa, foram incluídos entre 2011-2013 em dois ambulatórios de clínica geriátrica localizados na área metropolitana do Distrito Federal, Brasil, para avaliação dos determinantes dos transtornos circulatórios. Os ambulatórios clínicos foram o Serviço Geriátrico do Hospital da Universidade Católica de Brasília (HUCB) e o Centro Multidisciplinar do Idoso do Hospital da 
Universidade de Brasília (UnB), que recebem pacientes com características sóciodemográficas semelhantes.

Os pacientes admitidos no HUB foram encaminhados para o HUCB para a avaliação inicial e seguimento, em prol do desenvolvimento de protocolos clínicos e prestação de cuidados homogêneos, realizados pelo mesmo corpo clínico. O critério de inclusão consistiu na procura espontânea por cuidado preventivo para eventos circulatórios. Como critérios de exclusão para integrar estas análises: (i) doença autoimunológica, (ii) neoplasias de qualquer tipo, atuais ou passadas, (iii) doença pulmonar grave, (iv) doença renal sintomática ou assintomática crônica grave (depuração da creatinina de $25 \mathrm{ml} / \mathrm{min} / 1,73 \mathrm{~m}^{2}$ ), (v) infecções crônicas ou recorrentes, (vi) doença do fígado, definida por alterações nos testes de função bioquímica e /ou exames de imagem compatíveis com doença hepática grave, (vii) insuficiência cardíaca (fração de ejeção <50\%). Não houve busca ativa de pacientes com qualquer condição particular.

O projeto foi aprovado pelo Comitê de Ética e Pesquisa da Faculdade de Medicina da Universidade de Brasília, oficio 061/2011 (anexo I). Todos os participantes assinaram termo de consentimento livre e esclarecido (anexo II).

\subsection{AVALIAÇÃO CLÍNICA E BIOQUÍMICA}

A avaliação clínica de cada paciente constituiu na investigação da presença de diabetes mellitus e hipertensão arterial, uso contínuo de medicamentos para condições crônicas (anexo III), eventos vasculares prévios como infarto do miocárdio ou cerebrovascular, histórico familiar de doenças circulatórias e aspectos do estilo de vida como sedentarismo e hábito de fumar. 
Praticantes de exercícios físicos foram os que exibiram 30 minutos ou mais de exercícios de qualquer modalidade durante pelo menos quatro dias por semana, enquanto que o hábito de fumar foi definido como consumo de $>100$ cigarros ao longo da vida. Para a análise metabólica, as seguintes medidas foram obtidas de acordo com a bioquímica clínica de rotina: glicemia, hemoglobina glicosilada (HbA1c), insulina, colesterol total e frações, triglicérides, proteína C-reativa de alta sensibilidade, o índice HOMA, TSH e homocisteína. Na análise antropométrica foi avaliado o índice de massa corporal (IMC, em $\left.\mathrm{kg} / \mathrm{m}^{2}\right)$ e circunferência da cintura (cm).

\subsection{MEDIÇÕES DA CAMADA MÉDIO-INTIMAL CAROTÍDEA}

A espessura médio-intimal carotídea (EMIc) foi avaliada de forma não invasiva em todos os pacientes por ultrassom de alta resolução usando o equipamento Philips (modelo IE 33) com um transdutor linear de 3-9 MHz.

As medições da EMIc foram realizadas bilateralmente na parede da artéria carótida comum distal do bulbo e na origem da artéria carótida interna, por programa automatizado de detecção de bordas que é capaz de mostrar o valor máximo médio de 150 medições de qualquer seguimento de $10 \mathrm{~mm}$. O valor máximo da EMlc foi mensurado manualmente como a média de três medições independentes.

Todos os procedimentos clínicos e de avaliação das imagens foram realizados no HUCB e por mesmo observador.

\subsection{PAINEL INFLAMATÓRIO}


No momento da avaliação bioquímica, foi feita coleta de sangue total venoso e obtidas amostras de soro, que foram mantidas congeladas a $-80^{\circ} \mathrm{C}$ até serem descongeladas para a avaliação dos mediadores imunológicos.

As concentrações das citocinas foram obtidas por citometria de fluxo usando sistema multiplex com dois sets de imunoensaio baseado em esferas, a saber, Human Th1/Th2/TH17 e o Human Inflammatory, ambos produzidos pela empresa BD Biosciences ${ }^{\circledR}$ (San Diego, CA, USA).

Os procedimentos em laboratório seguiram os protocolos fornecidos pelo fabricante dos kits. Os kits juntos produziram medições para dez mediadores circulantes diferentes, a saber: interleucinas(IL)-16, IL-2, IL-4, IL-6, IL-8, IL-10, IL12p70, IL-17A, interferon gama (IFNo) e fator de necrose tumoral alfa (TNF-Ǔ).

Os padrões de citocinas liofilizadas e as amostras de soro foram processados juntamente, seguindo protocolo do fabricante, e os resultados obtidos utilizando o citômetro de fluxo BD FACSCalibur, canal FL4.

Os dados foram analisados usando o software FCAP software, versão 3.0 (BD Biosciences ${ }^{\circledR}$, San Diego, CA, USA). A curva padrão para cada citocina foi gerada utilizando padrão do mediador fornecido no kit. A concentração em cada soro foi determinada por interpolação a partir da curva padrão correspondente.

\subsection{ANÁLISE ESTATÍSTICA}

Para alcançar o objetivo de avaliar a ocorrência e a força da associação entre os níveis dos mediadores inflamatórios e as medidas do EMI da carótida, nossas análises estatísticas começaram com a obtenção de coeficientes de correlação entre estas variáveis e as variáveis (contínuas e categóricas) antropométricas, clínicas e bioquímicas com potencial efeito de confudimento no modelo principal. Para isso, a 
distribuição normal de todas as variáveis foi avaliada pelo teste de KolmogorovSmirnov e, sempre que necessário e possível, transformações logarítmicas foram aplicadas para se conseguir uma distribuição mais próxima da normalidade.

A associação entre as variáveis contínuas foi avaliada pelo teste de correlação de Pearson, enquanto que o envolvimento de uma variável categórica no modelo foi tratado usando o teste homólogo de Spearman. Para estas análises, a presença ou ausência de uma característica foi representada por 1 ou 0 , respectivamente. Sempre que uma interação foi notada, análises de correlação parcial foram realizadas utilizando o ajuste para as variáveis de confudimento ou condições. Para as análises de correlação, um valor de P (bicaudal) foi considerado significativo conforme o princípio de correção de Bonferroni para comparações múltiplas, segundo o qual se uma dada característica é testada contra $k$ variáveis independentes, o nível de significância deve ser ajustado à razão do $k$ de testes realizados (se $k=10$ testes, $\therefore \breve{U}=0,005$ ).

Além disso, a análise de variância foi utilizada para testar se as concentrações de citocinas logaritmicamente transformadas variaram entre os tercis das medidas do EMlc na amostra, com ajuste para co-variáveis, e P Ò 0,05 como limite de significância. Por fim, análise de regressão múltipla linear, método stepwise, foi realizada para avaliar em que medida as concentrações séricas dos mediadores inflamatórios explicavam a variabilidade dos valores de EMlc.

Todas as análises foram realizadas com programa Statistical Package for the Social Sciences (SPSS) para o Windows (versão 17.0). 


\section{RESULTADOS}

Foram analisados dados de 168 pacientes admitidos entre 2011 e 2013 quanto a aspectos clínicos e inflamatórios. A Tabela 1 apresenta as características antropométricas, cínicas e metabólicas da amostra. O perfil geral dos pacientes é compatível com distúrbios metabólicos limítrofes, com uma alta prevalência de casos de hipertensos, diabéticos e sedentários. Além disso, eventos cardiovasculares anteriores não foram raros na amostra como ilustrado por casos prévios de acidente vascular encefálico $(<10 \%)$, infarto agudo do miocárdio $(<5 \%)$ e doença arterial coronariana $(<2 \%)$.

Primeiramente, a análise de correlação dos níveis séricos brutos das citocinas e das medidas da EMI da carótida direita e esquerda por todas as características categóricas clínicas e de saúde não mostrou associação dos mediadores imunes e da arquitetura artéria com qualquer condição prévia de risco para o aparecimento de doenças cardiovasculares ou com aspectos que modulam ou tratam essas condições ( $p>0,005)$ (Tabela 2). A análise de correlação dos níveis logaritmicamente transformados do painel de citocinas e do EMI pelas características clínicas e bioquímicas contínuas demonstrou correlação e nível de significância entre o HDL-C com $\log _{10} \mathrm{lL} 16$ ( $\left.P<0,001\right)$, bem como com Log ${ }_{10} \mathrm{IL6}$ ( $\mathrm{P}$ $<0,004)$. No entanto, nenhuma destas últimas características mostrou correlação com os valores brutos do EMI da carótida direita e esquerda (Tabela 3). 
Tabela 1. Variáveis antropométricas, clínicas e metabólicas da amostra.

\begin{tabular}{|c|c|}
\hline Variáveis & Valor \\
\hline $\mathrm{n}$, sujeitos & 168 \\
\hline Homens, \% & 39,9 \\
\hline Idade, anos & $73,1 \pm 9,0$ \\
\hline IMC, kg.m² & $27,7 \pm 5,3$ \\
\hline Circunferência Abdominal, cm & $97,4 \pm 11,5$ \\
\hline Glicemia, mg.dl ${ }^{-1}$ & $103,2 \pm 27,7$ \\
\hline $\mathrm{HbA} 1 \mathrm{c}, \%$ & $5,9 \pm 1,0$ \\
\hline Insulina, mUl/mL & $9,3 \pm 10,0$ \\
\hline Índice HOMA index & $2,6 \pm 3,8$ \\
\hline DM2, \% & 22,6 \\
\hline Colesterol Total, mg.dll ${ }^{-1}$ & $193,4 \pm 39,9$ \\
\hline VLDL-c, mg.dl ${ }^{-1}$ & $29,8 \pm 15,2$ \\
\hline HDL-c, mg.dl ${ }^{-1}$ & $48,1 \pm 10,9$ \\
\hline LDL-c, mg.dl $^{-1}$ & $115,3 \pm 33,7$ \\
\hline Triglicérides, $\mathrm{mg} . \mathrm{dl}^{-1}$ & $141,2 \pm 64,7$ \\
\hline Pressão Sistólica, mm Hg & $134,8 \pm 19,5$ \\
\hline Pressão Diastólica, mm Hg & $80,6 \pm 11,1$ \\
\hline Hipertensos, \% & 77,4 \\
\hline $\mathrm{PCR}, \mathrm{mg} / \mathrm{dL}$ & $3,5 \pm 5,6$ \\
\hline TSH, mIU/L & $2,5 \pm 2,2$ \\
\hline Homocisteína, $\mu \mathrm{mol} / \mathrm{L}$ & $12,8 \pm 8,8$ \\
\hline AVE prévio, \% & 9,5 \\
\hline IAM prévio, \% & 4,2 \\
\hline DAC prévia, \% & 1,8 \\
\hline Sedentários, \% & 60,7 \\
\hline Fumantes, $\%$ & 36,9 \\
\hline EMlc direita, mm & $1,36 \pm 1,63$ \\
\hline EMlc esquerda, mm & $1,21 \pm 1,13$ \\
\hline
\end{tabular}

Dados expressos como média \pm desvio padrão para variáveis contínuas e frequências relativas para variáveis categóricas. $\mathrm{IMC}=$ índice de massa corporal; $\mathrm{HbA1 \textrm {C }}=$ hemoglobina glicada A1c; HOMA = Modelo de avaliação homeostático; DM2 = diabetes mellitus tipo 2; VLDL-C = lipoproteína de muita baixa densidade; $L D L-C$ = lipoproteína de baixa densidade; HDL-C = lipoproteína de alta densidade, $\mathrm{PCR}=$ Proteína $\mathrm{C}$ reativa; $\mathrm{TSH}=$ hormônio de estimulação tiroidiana; $\mathrm{AVE}=$ acidente vascular encefálico ; IAM $=$ infarto agudo do miocárdio; DAC = doença arterial coronariana; EMIc = espessura médio-intimal carotídea. 
Tabela 2. Análise de correlação dos valores brutos séricos das citocinas e das medidas absolutas de EMlc com características categóricas clínicas e de saúde na amostra à admissão.

\begin{tabular}{|c|c|c|c|c|c|c|c|c|c|c|}
\hline & \multicolumn{5}{|c|}{ Clínicos } & \multicolumn{5}{|c|}{ Saúde } \\
\hline & Sexo & DM2 & PAS & AVE & IAM & Fumante & Sedentário & $\begin{array}{c}\text { anti PAS } \\
\text { droga }\end{array}$ & $\begin{array}{l}\text { AntilipEMI } \\
\text { c droga }\end{array}$ & $\begin{array}{c}\text { anti DM2 } \\
\text { droga }\end{array}$ \\
\hline $\mathrm{IFN} \gamma$ & ,090;, 266 &,$- 070 ;, 382$ &, $107 ;, 182$ &, $066 ;, 410$ &,$- 056 ;, 487$ &, $155 ;, 055$ &, $076 ;, 344$ & ,053;,511 & ,074;,361 &,$- 058 ;, 476$ \\
\hline IL16 &,$- 013 ;, 869$ &, $017 ;, 834$ &,$- 077 ;, 326$ &,$- 091 ;, 250$ &, $016 ;, 844$ &, $082 ;, 304$ &, $025 ;, 751$ &,$- 089 ;, 259$ & ,001; 992 &, $035 ;, 654$ \\
\hline IL2 &, $061 ;, 450$ &,$- 054 ;, 503$ &, $057 ;, 477$ & ,096; ,231 &, $056 ;, 487$ &, $122 ;, 131$ & ,099; ,217 & ,032;,692 &,$- 000 ;, 997$ &,$- 097 ;, 229$ \\
\hline IL4 &, $022 ;, 783$ &,$- 031 ;, 697$ &, $049 ;, 543$ & ,001; ,991 &,$- 013 ;, 868$ &, $102 ;, 208$ &, $083 ;, 304$ &, $066 ;, 413$ & ,068; ,402 &,$- 051 ;, 526$ \\
\hline IL6 & ,087; ,270 &,$- 135 ;, 085$ &,$- 155 ;, 048$ &,$- 071 ;, 369$ &, $127 ;, 107$ &, $002 ;, 981$ & ,089; ,257 &,$- 153 ;, 052$ &, $145 ;, 064$ &,$- 039 ;, 620$ \\
\hline IL8 &,$- 050 ;, 523$ &, $032 ;, 683$ &,$- 025 ;, 755$ &,$- 057 ;, 470$ &, $123 ;, 118$ &,$- 003 ;, 968$ &, $025 ;, 750$ &,$- 001 ;, 991$ &, $002 ;, 983$ & ,066; ,399 \\
\hline IL10 &, $123 ;, 118$ &, $038 ;, 630$ &,$- 066 ;, 401$ &,$- 101 ;, 200$ & ,002; ,980 &, $003 ;, 972$ &, $044 ;, 575$ & ,004; ,963 &,$- 126 ;, 109$ &, $061 ;, 439$ \\
\hline IL12.p70 &,$- 033 ;, 680$ &,$- 023 ;, 766$ &,$- 069 ;, 384$ &,$- 162 ;, 022$ &, $033 ;, 675$ &,$- 011 ;, 890$ &,$- 002 ;, 978$ &,$- 054 ;, 492$ &,$- 200 ;, 011$ & ,007; ,926 \\
\hline IL17a &,$- 020 ;, 801$ & ,010; ,905 &, $118 ;, 143$ & ,009; ,910 &,$- 056 ;, 484$ &, $104 ;, 198$ &, $128 ;, 111$ &, $108 ;, 181$ &, $073 ;, 362$ &,$- 070 ;, 383$ \\
\hline TNFŬ &, $120 ;, 128$ &,$- 084 ;, 289$ &,$- 044 ;, 579$ &,$- 144 ;, 066$ &, $013 ;, 869$ &, $110 ;, 164$ &, $135 ;, 085$ &, $014 ;, 854$ &,$- 027 ;, 728$ &,$- 022 ;, 776$ \\
\hline EMIc direita &,$- 037 ;, 708$ & ,091; ,358 &, $116 ;, 245$ &, $180 ;, 068$ & ,089; ,370 &, $077 ;, 044$ &, $108 ;, 277$ & ,220;,026 &, $076 ;, 443$ &, $062 ;, 537$ \\
\hline EMIc esquerda &,$- 010 ;, 922$ &,$- 034 ;, 745$ &,$- 049 ;, 637$ & ,246; ,016 &, $098 ;, 341$ & ,090; ,389 &, $245 ;, 016$ &,$- 050 ;, 629$ &, $037 ;, 724$ &,$- 060 ;, 559$ \\
\hline
\end{tabular}

Resultados de teste de correlação de Spearman. Dados expressos em índice de correlação e de significância $(r, P)$. DM2 = diabetes mellitus tipo 2; HAS = hipertensão arterial sistêmica ; IAM = infarto agudo do miocárdio; IFNo = interferon gama; IL = interleucina ; TNFŬ = fator de necrose tumoral alfa; EMlc = espessura médio-intimal carotídea. 
Tabela 3. Análise de correlação dos níveis séricos logaritmicamente transformados da citocinas e das medidas absolutas de EMlc com características clínicas e bioquímicas de natureza contínua na amostra à admissão.

\begin{tabular}{|c|c|c|c|c|c|c|c|c|c|c|}
\hline & \multicolumn{4}{|c|}{ Clínicos } & \multicolumn{6}{|c|}{ Bioquímicos } \\
\hline & Idade & $\mathrm{CA}$ & PAS & PAD & CT & VLDL-c & HDL-c & TGL & HOMA & HbAlc \\
\hline $\log _{10}$ IFN $\gamma$ &, $046 ;, 568$ &,$- 011 ;, 894$ & ,098; ,223 & ,087; ,282 &,$- 022 ;, 785$ &, $063 ;, 438$ &,$- 115 ;, 153$ & ,086; ,288 &, $058 ;, 477$ &,$- 030 ;, 716$ \\
\hline $\log _{10}$ IL16 & ,091; ,251 &, $012 ;, 880$ &, $078 ;, 325$ &, $037 ;, 641$ &, $154 ;, 050$ &, $076 ;, 339$ & ,247; ,001 & ,094; , 232 &, $029 ;, 717$ &, $015 ;, 851$ \\
\hline $\log _{10} \mathrm{IL} 2$ &,$- 044 ;, 587$ &, $040 ;, 623$ & ,009; ,914 & ,067; ,404 &,$- 006 ;, 942$ & ,006; ,945 & ,010; ,902 &, $063 ;, 432$ &,$- 006 ;, 939$ &,$- 110 ;, 177$ \\
\hline $\log _{10}$ IL4 &,$- 069 ;, 392$ &, $061 ;, 456$ &, $119 ;, 138$ &, $155 ;, 053$ &,$- 002 ;, 984$ & ,102; ,207 &,$- 037 ;, 645$ &, $122 ;, 128$ &, $025 ;, 758$ &,$- 049 ;, 151$ \\
\hline $\log _{10}$ IL6 &, $073 ;, 353$ &,$- 089 ;, 262$ &,$- 104 ;, 185$ &,$- 074 ;, 348$ &, $069 ;, 384$ &,$- 136 ;, 084$ & ,218;,004 &,$- 132 ;, 094$ &,$- 177 ;, 025$ &,$- 167 ;, 036$ \\
\hline $\log _{10} \mathrm{IL} 8$ & ,138; ,078 &,$- 002 ;, 983$ & ,034;,669 &,$- 032 ;, 688$ &, $062 ;, 435$ &,$- 002 ;, 979$ &, $110 ;, 164$ &,$- 022 ;, 779$ &, $017 ;, 830$ &,$- 014 ;, 860$ \\
\hline $\log _{10}$ IL10 &,$- 020 ;, 800$ &, $014 ;, 862$ &, $024 ;, 765$ &, $043 ;, 587$ &, $182 ;, 020$ &, $089 ;, 258$ &, $127 ;, 108$ &, $140 ;, 075$ &, $064 ;, 426$ &, $085 ;, 290$ \\
\hline $\log _{10}$ IL12.p70 &,$- 111 ;, 080$ &, $024 ;, 763$ &, $070 ;, 378$ &, $062 ;, 429$ &, $150 ;, 055$ & ,092; ,246 & ,087; ,270 &, $095 ;, 226$ & ,001; ,993 &, $072 ;, 369$ \\
\hline $\log _{10} \operatorname{IL} 17 \mathrm{a}$ &, $056 ;, 491$ &,$- 062 ;, 443$ &, $115 ;, 154$ &, $132 ;, 104$ &,$- 059 ;, 466$ &, $055 ;, 501$ &,$- 042 ;, 606$ & ,087; ,279 &, $006 ;, 940$ & ,006; ,939 \\
\hline $\log _{10}$ TNFŬ & ,077; ,328 &, $044 ;, 583$ & ,032;,689 &, $015 ;, 846$ &, $108 ;, 177$ &, $048 ;, 544$ & ,179; ,022 &, $071 ;, 365$ &,$- 063 ;, 430$ &,$- 068 ;, 399$ \\
\hline EMIc direita & ,196; ,048 &,$- 105 ;, 293$ &, $014 ;, 886$ &, $028 ;, 781$ &,$- 094 ;, 344$ &,$- 084 ;, 400$ &, $094 ;, 346$ &,$- 067 ;, 502$ &,$- 013 ;, 902$ &,$- 061 ;, 548$ \\
\hline EMIc esquerda & ,229; ,025 &,$- 094 ;, 365$ &, $032 ;, 757$ &, $066 ;, 521$ &, $054 ;, 600$ &,$- 024 ;, 813$ &, $116 ;, 262$ &,$- 011 ;, 917$ &, $078 ;, 459$ &,$- 049 ;, 641$ \\
\hline
\end{tabular}

Resultados de teste de correlação de Pearson. Dados expressos pelo índice de correlação e nível de significância $(r, P)$. CA = circunferência abdominal; PAS = pressão arterial sistólica; PAD = pressão arterial diastólica; $C T$ = colesterol total; VLDL-c = lipoproteína de muita baixa densidade; HDL-C = lipoproteína de alta densidade; HOMA = Modelo de avaliação homeostático; HbA1c = hemoglobina glicada A1c; Log = dado logaritmicamente transformado; IFNŬ = interferon gama; IL = interleucina ; TNFŬ = fator de necrose tumoral alfa; EMlc = espessura médio-intimal carotídea. 
$\mathrm{Na}$ análise de correlação entre os níveis circulatórios logaritmicamente transformados das citocinas e dos valores brutos das medidas da EMlc, os mediadores imunes IL16, IL6, IL8, IL10 e TNFŬ demonstraram nível de significância de associação positiva (P Ò 0.005) com as medidas do EMI da carótida esquerdo, mas não com o direito. Os outros mediadores imunes logaritmicamente transformados (IFNo, IL2, IL4, IL12p70 e IL17a) não apresentaram associação com nenhuma das medidas da EMIc.

Tabela 4. Análise de correlação entre os níveis circulatórios logaritmicamente transformados das citocinas e valores brutos das medidas da EMlc na amostra à admissão.

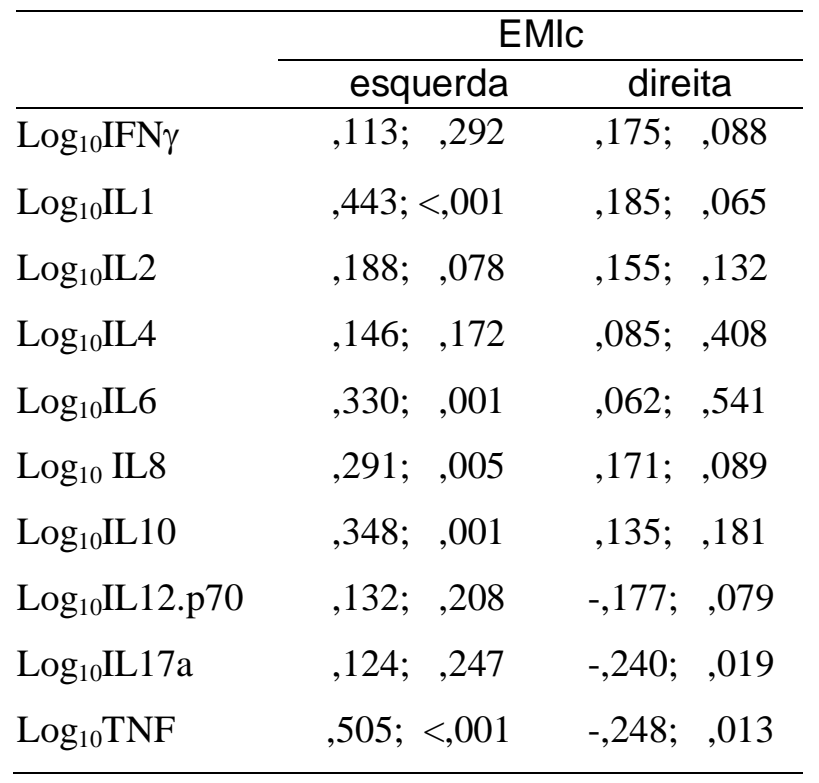

Resultados de teste de correlação de Pearson. Dados expressos como índice de correlação e nível de significância $(r, P)$. Log = dado logaritmicamente transformado; IFN $=$ interferon gama; IL = interleucina; TNFŬ = fator de necrose tumoral alfa; EMlc = espessura médiointimal carotídea.

Assim, a comparação dos níveis circulatórios logaritmicamente transformados das citocinas em todos os indivíduos agrupados em tercis de aumento com o 
espessamento médio-intimal da carótida esquerda confirma correlação positiva com os níveis de TNFǓ e IL16, ajustados para HDL-c (Figura 1), enquanto IL6, IL8 e IL10 não alcançaram diferença significativa em todos os tercis com esta abordagem. E a regressão multivariada passo a passo confirmou que $\log _{10}$ TNFŬ foi a variável mais preditiva do modelo $\left(\mathrm{R}^{2}=0.209\right)$, o que acrescida dos $\log _{10} \mathrm{IL} 16$ e $\log _{10} \mathrm{IL6}$ nesta ordem responderam por 29,5\% da variância das medidas da EMIc.
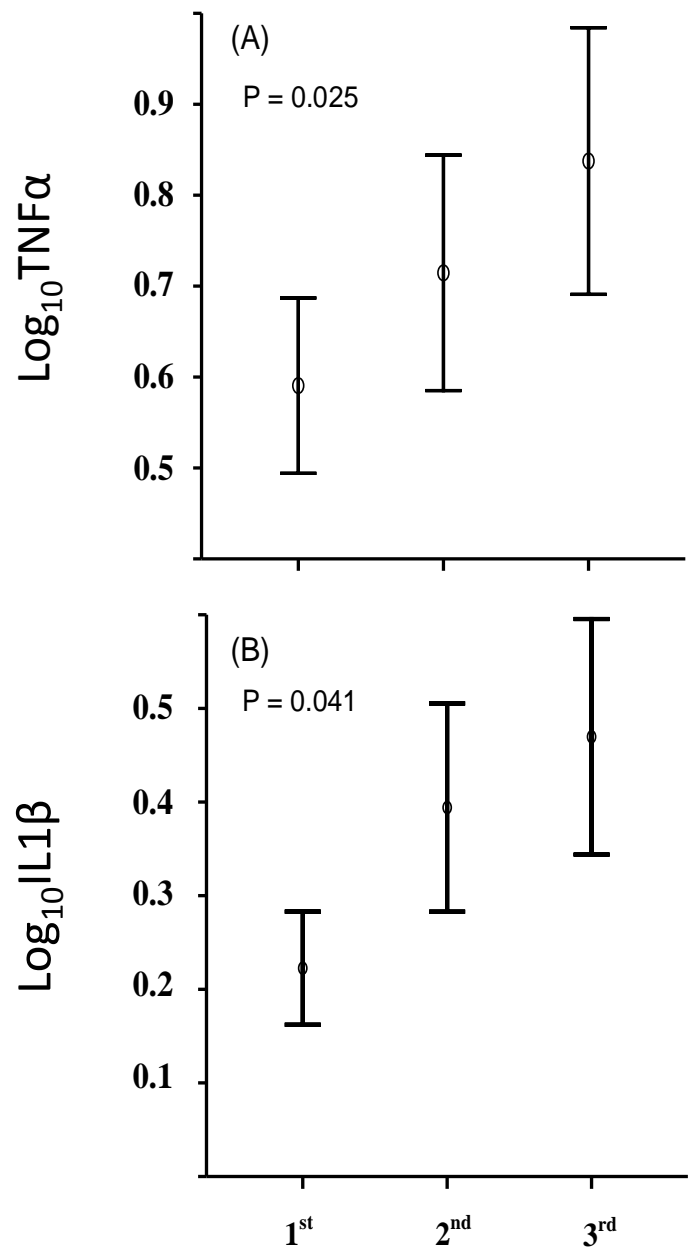

Tercis de EMIc esquerda

Figura 1. Comparação entre os níveis circulantes logaritmicamente transformados de TNFŬ (A) e IL16 (B) de indivíduos agrupados em tercis de espessura médio- 
intimal da artéria carotídea esquerda. Significância verificada por ANCOVA ajustada para o HDL-c. As barras verticais representam o intervalo de um erro padrão. 


\section{DISCUSSÃO}

Procuramos neste trabalho investigar a relação das características clínicas e bioquímicas, das medidas de saúde e das características inflamatórias com um aspecto importante da morfologia carotídea, e encontramos que os níveis logaritmicamente transformados das citocinas IL16, IL6, IL8, IL10 e TNFŬ foram independentemente correlacionados com a medida da EMI da carótida em seu ramo esquerdo. Nossos resultados reforçam o posicionando que sugere o envolvimento de um conjunto de citocinas circulantes pró-inflamatórios no processo arteriosclerótico deste leito vascular encefálico. Curiosamente, não encontramos correlação com as outras variáveis clínicas e bioquímicas ou de saúde avaliadas aqui com a morfologia carotídea.

Ao apontar para uma possível participação das citocinas no processo aterosclerótico carotídeo, nossos achados vão ao encontro de estudos epidemiológicos em que pacientes com doenças inflamatórias crônicas, como artrite reumatoide (AR), apresentam risco significativamente maior de desenvolver doença arterial coronariana em comparação com a população em geral ${ }^{13}$, apresentando também uma maior espessura das paredes carotídeas ${ }^{14}$. Ademais, corroboram também o fato de mediadores inflamatórios como TNFŬ e IL6 (este último como resultado usual da produção de TNFŬ) estarem significativamente associados com a aterosclerose prematura na artéria carótida ${ }^{15 ;}{ }^{16}$, independentemente de fatores de risco tradicionais ${ }^{17 ;}$ 18. Pelo exposto, não causa estranheza de que o uso de antagonista de TNF-Ǔ melhorou a função endotelial e produziu redução de EMI carotídea de pacientes com $\mathrm{AR}^{19 ; 20 ; 21}$.

Evidências de que IL16 desempenha um papel importante na aterosclerose também estão disponíveis na literatura. Sendo sintetizada pelas células endoteliais, 
células musculares lisas vasculares (VSCM) e macrófagos, IL16 pode induzir a produção de outras citocinas, ativação de células endoteliais, aumento da permeabilidade vascular e proliferação das próprias VSMC ${ }^{22 ; 23}$. A falta de IL16 em ratos deficientes em ApoE reduz lesões ateroscleróticas, ao passo que sua expressão aumentada desencadeou a expressão aumentada da molécula de adesão intercelular-1 (ICAM-1) e da proteína quimiotáctica de monócitos-1 (MCP-1) no endotélio de aorta, possivelmente resultando em aumento de recrutamento de monócitos/macrófagos para a camada íntima ${ }^{24}$.

No conjunto de citocinas que apresentaram correlação com a EMI da carótida, IL10 consiste na única que apresenta propriedades anti-inflamatórias. O aumento da produção endógena de IL10 reduz a extensão e/ou a gravidade de aterosclerose e estabiliza fenótipos ateroscleróticos vulneráveis ${ }^{25 ;} 26$. IL10 desempenha um sistema de controle importante, havendo relatos de que LDL-c oxidado induz sua produção, o que diminuiria a liberação de citocinas inflamatórias, desenvolvendo assim funções imunoregulatórias de modo a impedir a resposta inflamatória excessiva e processos fibróticos associados ${ }^{27}$. Corroborando com a ideia de seu papel na modulação da resposta inflamatória, modelo de camundongos deficientes de medula demostrou que a expressão de IL10 por leucócitos preveniu o desenvolvimento de lesões ateroscleróticas avançadas e desempenhou papel crítico na modulação da composição celular e de colágeno da placa ${ }^{28}$. Portanto, os níveis aumentados de IL10 entre portadores de maior EMlc pode ser simplesmente o resultado de um mecanismo compensatório em resposta à elevação sistêmica na sinalização próinflamatória.

A artéria carótida comum ( $A C C$ ) esquerda tem se mostrado ser mais vulnerável à aterosclerose quando comparada ao ramo direito, apresentando maior 
EMI, rigidez e número de placas ateromatosas ${ }^{29}{ }^{30}$. A anatomia do sistema circulatório pode, ao menos em parte, explicar esta etiologia assimétrica de achados patológicos. Normalmente, a ACC esquerda tem origem no arco da aorta enquanto a ACC direita na artéria inominada ${ }^{31}$. Esta diferença anatômica expõe a ACC esquerda a uma maior intensidade de fluxo e pressão (e, consequentemente, maior força de cisalhamento) a partir do arco aórtico, uma vez que a ACC direita está submetida a menor fluxo de sangue pela aorta ascendente ${ }^{30}$. Neste cenário, é razoável supor que as lesões vasculares desencadeadas por uma maior pressão hidrostática pode ser intensificadas de uma forma proporcional ao ambiente próinflamatório sistêmico, fator que pode não ser um importante modulador fisiopatológico de lesões em territórios vasculares menos turbulentos.

Considerados todos os aspectos acima, nosso estudo encontra-se de acordo com evidências robustas presentes na literatura de que o desenvolvimento de doenças crônicas relacionadas com a idade é acompanhado por um estado de baixo grau de inflamação sistêmica e subclínica, denominado inflammaging. Para os autores, a revelação de que os mediadores pró-inflamatórios IL16, IL6, IL8 e TNFŬ figuram como possíveis contribuintes para o fenótipo da aterosclerose proporciona uma ilustração deste fenômeno ${ }^{32 ; 33}$. Afinal, elevações subclínicas dos níveis de citocinas circulantes figuram como importante fator de risco para morbidade e mortalidade em idosos, independentemente de outros fatores relacionados a estilo de vida ${ }^{34}$.

Mas nosso estudo apresenta limitações, a despeito de todos os cuidados metodológicos e estatísticos empregados. O desenho transversal impede determinar a causalidade entre as variáveis. Além disso, a determinação do número e do perfil morfológico das placas de ateroma encontrava-se além de nossa capacidade 
técnica para avaliação. Por fim, uma possível interferência por variáveis nutricionais ou de estilo de vida não foi considerada. 


\section{CONCLUSÃo}

Demonstramos que os níveis logaritmicamente transformados de IL16, IL6, IL8, IL10 e TNF-Ŭ correlacionaram-se independentemente com medidas de espessura médio-intimal da artéria carótida esquerda (porém não com a equivalente direita), com as associações mais fortes obtidas com TNFŬ, IL16 e IL6, nesta ordem. Nosso trabalho sugere que seja possível uma contribuição de mediadores inflamatórios ao processo aterosclerótico em território cerebrovascular específico, e que os achados provavelmente refletem o fenômeno do inflammaging. 


\section{REFERÊNCIAS}

MALLAT, Z. ET AL. THE ROLE OF ADAPTIVE T CELL IMMUNITY IN ATHEROSCLEROSIS. J LIPID RES, V. 50 SUPPL, P. S364-9, APR 2009. ISSN 0022-2275 (PRINT) 0022-2275 (LINKING). DISPONÍVEL EM: <HTTP:/NWWW.NCBI.NLM.NIH.GOV/PUBMED/19050311 >.

MORRIS, P. B. ET AL. REVIEW OF CLINICAL PRACTICE GUIDELINES FOR THE MANAGEMENT OF LDL-RELATED RISK. JOURNAL OF THE AMERICAN COLLEGE OF CARDIOLOGY, V. 64, N. 2, P. 196-206, 7/15/ 2014. ISSN 0735-1097. DISPONÍVEL

EM:

<HTTP:/WWW.SCIENCEDIRECT.COM/SCIENCE/ARTICLE/PII/S073510971402591 $1>$.

HWANG, M. H.; KIM, S. TYPE 2 DIABETES: ENDOTHELIAL DYSFUNCTION AND EXERCISE. J EXERC NUTRITION BIOCHEM, V. 18, N. 3, P. 239-47, SEP 2014. ISSN 2233-6834 (PRINT) 2233-6834 (LINKING). DISPONÍVEL EM: < HTTP:/WWW.NCBI.NLM.NIH.GOV/PUBMED/25566460 >.

BERLINER, J. A. ET AL. ATHEROSCLEROSIS: BASIC MECHANISMS. OXIDATION, INFLAMMATION, AND GENETICS. CIRCULATION, V. 91, N. 9, P. 2488-96, MAY 1 1995. ISSN 0009-7322 (PRINT) 0009-7322 (LINKING).

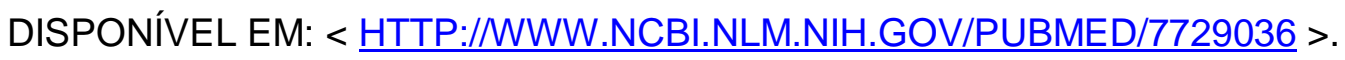

LIBBY, P.; RIDKER, P. M.; HANSSON, G. K. PROGRESS AND CHALLENGES IN TRANSLATING THE BIOLOGY OF ATHEROSCLEROSIS. NATURE, V. 473, N. 7347, P. 317-25, MAY 19 2011. ISSN 1476-4687 (ELECTRONIC) 0028-0836 (LINKING). DISPONÍVEL EM:

HTTP:/WWW.NCBI.NLM.NIH.GOV/PUBMED/21593864 >.

STEIN, J. H. ET AL. USE OF CAROTID ULTRASOUND TO IDENTIFY SUBCLINICAL VASCULAR DISEASE AND EVALUATE CARDIOVASCULAR DISEASE RISK: A CONSENSUS STATEMENT FROM THE AMERICAN SOCIETY OF ECHOCARDIOGRAPHY CAROTID INTIMA-MEDIA THICKNESS TASK FORCE. ENDORSED BY THE SOCIETY FOR VASCULAR MEDICINE. J AM SOC ECHOCARDIOGR, V. 21, N. 2, P. 93-111; QUIZ 189-90, FEB 2008. ISSN 1097-6795 (ELECTRONIC) 0894-7317 (LINKING). DISPONÍVEL EM: < HTTP:/NWW.NCBI.NLM.NIH.GOV/PUBMED/18261694 >. 
PETERS, S. A. ET AL. IMPROVEMENTS IN RISK STRATIFICATION FOR THE OCCURRENCE OF CARDIOVASCULAR DISEASE BY IMAGING SUBCLINICAL ATHEROSCLEROSIS: A SYSTEMATIC REVIEW. HEART, V. 98, N. 3, P. 177-84, FEB 2012. ISSN 1468-201X (ELECTRONIC) 1355-6037 (LINKING). DISPONÍVEL EM: < HTTP:/NWWW.NCBI.NLM.NIH.GOV/PUBMED/22095617 > .

VAN DEN OORD, S. C. ET AL. CAROTID INTIMA-MEDIA THICKNESS FOR CARDIOVASCULAR RISK ASSESSMENT: SYSTEMATIC REVIEW AND METAANALYSIS. ATHEROSCLEROSIS, V. 228, N. 1, P. 1-11, MAY 2013. ISSN 18791484 (ELECTRONIC) 0021-9150 (LINKING). DISPONÍVEL EM: < HTTP:/NWW.NCBI.NLM.NIH.GOV/PUBMED/23395523 >.

LORENZ, M. W. ET AL. PREDICTION OF CLINICAL CARDIOVASCULAR EVENTS WITH CAROTID INTIMA-MEDIA THICKNESS: A SYSTEMATIC REVIEW AND META-ANALYSIS. CIRCULATION, V. 115, N. 4, P. 459-67, JAN 30 2007. ISSN 1524-4539 (ELECTRONIC) 0009-7322 (LINKING). DISPONÍVEL EM: < HTTP:/NWW.NCBI.NLM.NIH.GOV/PUBMED/17242284 >.

YEBOAH, J. ET AL. MEDIATION OF CARDIOVASCULAR RISK FACTOR EFFECTS THROUGH SUBCLINICAL VASCULAR DISEASE: THE MULTI-ETHNIC STUDY OF ATHEROSCLEROSIS. ARTERIOSCLER THROMB VASC BIOL, V. 34, N. 8, P. 1778-83, AUG 2014. ISSN 1524-4636 (ELECTRONIC) 1079-5642 (LINKING). DISPONÍVEL EM:

HTTP:/WWW.NCBI.NLM.NIH.GOV/PUBMED/24876350 >.

LIAO, S. Q. ET AL. THE ASSOCIATION BETWEEN LEUKOARAIOSIS AND CAROTID ATHEROSCLEROSIS: A SYSTEMATIC REVIEW AND META-ANALYSIS. INT J NEUROSCI, AUG 28 2014. ISSN 1563-5279 (ELECTRONIC) 0020-7454 (LINKING).

DARABIAN, S. ET AL. THE ROLE OF CAROTID INTIMAL THICKNESS TESTING AND RISK PREDICTION IN THE DEVELOPMENT OF CORONARY ATHEROSCLEROSIS. CURR ATHEROSCLER REP, V. 15, N. 3, P. 306, MAR 2013. ISSN 1534-6242 (ELECTRONIC) 1523-3804 (LINKING). DISPONIVEL EM: < HTTP:/NWW.NCBI.NLM.NIH.GOV/PUBMED/23328906 >. 
MED, V. 121, N. 10 SUPPL 1, P. S15-20, OCT 2008. ISSN 1555-7162 (ELECTRONIC) 0002-9343 (LINKING). DISPONÍVEL EM: < HTTP:/WWW.NCBI.NLM.NIH.GOV/PUBMED/18926165 >.

VAN SIJL, A. M. ET AL. CAROTID INTIMA MEDIA THICKNESS IN RHEUMATOID ARTHRITIS AS COMPARED TO CONTROL SUBJECTS: A META-ANALYSIS. SEMIN ARTHRITIS RHEUM, V. 40, N. 5, P. 389-97, APR 2011. ISSN 1532-866X (ELECTRONIC) 0049-0172 (LINKING). DISPONÍVEL EM: < HTTP:/WWW.NCBI.NLM.NIH.GOV/PUBMED/20889191 >.

OKAZAKI, S. ET AL. ASSOCIATION OF INTERLEUKIN-6 WITH THE PROGRESSION OF CAROTID ATHEROSCLEROSIS: A 9-YEAR FOLLOW-UP STUDY. STROKE, V. 45, N. 10, P. 2924-9, OCT 2014. ISSN 1524-4628 (ELECTRONIC) 0039-2499 (LINKING). DISPONÍVEL EM: < HTTP:/WWW.NCBI.NLM.NIH.GOV/PUBMED/25139874 >.

SKOOG, T. ET AL. PLASMA TUMOUR NECROSIS FACTOR-ALPHA AND EARLY CAROTID ATHEROSCLEROSIS IN HEALTHY MIDDLE-AGED MEN. EUR HEART J, V. 23, N. 5, P. 376-83, MAR 2002. ISSN 0195-668X (PRINT) 0195-668X (LINKING). DISPONÍVEL EM: < HTTP://WWW.NCBI.NLM.NIH.GOV/PUBMED/11846495 >.

RHO, Y. H. ET AL. INFLAMMATORY MEDIATORS AND PREMATURE CORONARY ATHEROSCLEROSIS IN RHEUMATOID ARTHRITIS. ARTHRITIS RHEUM, V. 61, N. 11, P. 1580-5, NOV 15 2009. ISSN 0004-3591 (PRINT) 00043591 (LINKING). DISPONIVEL EM: < HTTP:/WWW.NCBI.NLM.NIH.GOV/PUBMED/19877084 >.

COLLABORATION, I. R. G. C. E. R. F. ET AL. INTERLEUKIN-6 RECEPTOR PATHWAYS IN CORONARY HEART DISEASE: A COLLABORATIVE METAANALYSIS OF 82 STUDIES. LANCET, V. 379, N. 9822, P. 1205-13, MAR 312012. ISSN 1474-547X (ELECTRONIC) 0140-6736 (LINKING). DISPONÍVEL EM: < HTTP:/WWW.NCBI.NLM.NIH.GOV/PUBMED/22421339 >.

NARANJO, A. ET AL. CARDIOVASCULAR DISEASE IN PATIENTS WITH RHEUMATOID ARTHRITIS: RESULTS FROM THE QUEST-RA STUDY. ARTHRITIS RES THER, V. 10, N. 2, P. R30, 2008. ISSN 1478-6362 (ELECTRONIC) 1478-6354 (LINKING). DISPONÍVEL EM:

HTTP:/WWW.NCBI.NLM.NIH.GOV/PUBMED/18325087 >. 
HURLIMANN, D. ET AL. ANTI-TUMOR NECROSIS FACTOR-ALPHA TREATMENT IMPROVES ENDOTHELIAL FUNCTION IN PATIENTS WITH RHEUMATOID ARTHRITIS. CIRCULATION, V. 106, N. 17, P. 2184-7, OCT 22 2002. ISSN 15244539 (ELECTRONIC) 0009-7322 (LINKING). DISPONÍVEL EM: < HTTP:/WWW.NCBI.NLM.NIH.GOV/PUBMED/12390945 >.

DEL PORTO, F. ET AL. RESPONSE TO ANTI-TUMOUR NECROSIS FACTOR ALPHA BLOCKADE IS ASSOCIATED WITH REDUCTION OF CAROTID INTIMAMEDIA THICKNESS IN PATIENTS WITH ACTIVE RHEUMATOID ARTHRITIS. RHEUMATOLOGY (OXFORD), V. 46, N. 7, P. 1111-5, JUL 2007. ISSN 1462-0324 (PRINT) 1462-0324

(LINKING).

DISPONÍVEL

EM:

HTTP:/WWW.NCBI.NLM.NIH.GOV/PUBMED/17449484 >.

FEARON, W. F.; FEARON, D. T. INFLAMMATION AND CARDIOVASCULAR DISEASE: ROLE OF THE INTERLEUKIN-1 RECEPTOR ANTAGONIST. CIRCULATION, V. 117, N. 20, P. 2577-9, MAY 20 2008. ISSN 1524-4539 (ELECTRONIC) 0009-7322 (LINKING). DISPONÍVEL EM: < HTTP:/WWW.NCBI.NLM.NIH.GOV/PUBMED/18490534 >.

GALEA, J. ET AL. INTERLEUKIN-1 BETA IN CORONARY ARTERIES OF PATIENTS WITH ISCHEMIC HEART DISEASE. ARTERIOSCLER THROMB VASC BIOL, V. 16, N. 8, P. 1000-6, AUG 1996. ISSN 1079-5642 (PRINT) 1079-5642 (LINKING). DISPONÍVEL EM:

HTTP:/MWW.NCBI.NLM.NIH.GOV/PUBMED/8696938 >.

KIRII, H. ET AL. LACK OF INTERLEUKIN-1BETA DECREASES THE SEVERITY OF ATHEROSCLEROSIS IN APOE-DEFICIENT MICE. ARTERIOSCLER THROMB VASC BIOL, V. 23, N. 4, P. 656-60, APR 1 2003. ISSN 1524-4636 (ELECTRONIC) 1079-5642 (LINKING). DISPONÍVEL EM: HTTP:/WWW.NCBI.NLM.NIH.GOV/PUBMED/12615675 >.

TZIAKAS, D. N. ET AL. ANTI-INFLAMMATORY CYTOKINE PROFILE IN ACUTE CORONARY SYNDROMES: BEHAVIOR OF INTERLEUKIN-10 IN ASSOCIATION WITH SERUM METALLOPROTEINASES AND PROINFLAMMATORY CYTOKINES. INT J CARDIOL, V. 92, N. 2-3, P. 169-75, DEC 2003. ISSN 0167-5273 (PRINT) 0167-5273 (LINKING). DISPONÍVEL EM: HTTP:/WWW.NCBI.NLM.NIH.GOV/PUBMED/14659849 >. 
MORAITIS, A. G. ET AL. ELEVATED INTERLEUKIN-10: A NEW CAUSE OF DYSLIPIDEMIA LEADING TO SEVERE HDL DEFICIENCY. J CLIN LIPIDOL, V. 9, N. 1, P. 81-90, JAN-FEB 2015. ISSN 1933-2874 (PRINT) 1876-4789 (LINKING). DISPONÍVEL EM: <

BENITEZ, S. ET AL. PRO-INFLAMMATORY ACTION OF LDL(-) ON MONONUCLEAR CELLS IS COUNTERACTED BY INCREASED IL10 PRODUCTION. BIOCHIM BIOPHYS ACTA, V. 1771, N. 5, P. 613-22, MAY 2007. ISSN 0006-3002 (PRINT) 0006-3002 (LINKING). DISPONÍVEL EM: < HTTP:/MWW.NCBI.NLM.NIH.GOV/PUBMED/17442617 >.

POTTEAUX, S. ET AL. LEUKOCYTE-DERIVED INTERLEUKIN 10 IS REQUIRED FOR PROTECTION AGAINST ATHEROSCLEROSIS IN LOW-DENSITY LIPOPROTEIN RECEPTOR KNOCKOUT MICE. ARTERIOSCLER THROMB VASC BIOL, V. 24, N. 8, P. 1474-8, AUG 2004. ISSN 1524-4636 (ELECTRONIC) 10795642 (LINKING). DISPONÍVEL EM: < HTTP:/WWW.NCBI.NLM.NIH.GOV/PUBMED/15178562 >.

YUAN, C. ET AL. CUMULATIVE EFFECTS OF HYPERTENSION, DYSLIPIDEMIA, AND CHRONIC KIDNEY DISEASE ON CAROTID ATHEROSCLEROSIS IN CHINESE PATIENTS WITH TYPE 2 DIABETES MELLITUS. J DIABETES RES, V. 2014, P. 179686, 2014. ISSN 2314-6753 (ELECTRONIC). DISPONÍVEL EM: < HTTP:/WWW.NCBI.NLM.NIH.GOV/PUBMED/24860832 >.

LUO, X. ET AL. DIFFERENCES IN LEFT AND RIGHT CAROTID INTIMA-MEDIA THICKNESS AND THE ASSOCIATED RISK FACTORS. CLIN RADIOL, V. 66, N. 5, P. 393-8, MAY 2011. ISSN 1365-229X (ELECTRONIC) 0009-9260 (LINKING). DISPONÍVEL EM: < HTTP://WWW.NCBI.NLM.NIH.GOV/PUBMED/21324442 >.

LAYTON, K. F. ET AL. BOVINE AORTIC ARCH VARIANT IN HUMANS: CLARIFICATION OF A COMMON MISNOMER. AJNR AM J NEURORADIOL, V. 27, N. 7, P. 1541-2, AUG 2006. ISSN 0195-6108 (PRINT) 0195-6108 (LINKING). DISPONÍVEL EM: < HTTP://WWW.NCBI.NLM.NIH.GOV/PUBMED/16908576 >.

MICHAUD, M. ET AL. PROINFLAMMATORY CYTOKINES, AGING, AND AGERELATED DISEASES. J AM MED DIR ASSOC, V. 14, N. 12, P. 877-82, DEC 2013. ISSN 1538-9375 (ELECTRONIC) 1525-8610 (LINKING). DISPONÍVEL EM: < HTTP:/WWW.NCBI.NLM.NIH.GOV/PUBMED/23792036 >. 
FRANCESCHI, C. ET AL. INFLAMM-AGING. AN EVOLUTIONARY PERSPECTIVE ON IMMUNOSENESCENCE. ANN N Y ACAD SCI, V. 908, P. 244-54, JUN 2000. ISSN $0077-8923$ (PRINT) $0077-8923$ (LINKING). DISPONÍVEL EM: < HTTP:/NWW.NCBI.NLM.NIH.GOV/PUBMED/10911963 >.

BRÜÜNSGAARD, H.; PEDERSEN, B. K. AGE-RELATED INFLAMMATORY CYTOKINES AND DISEASE. IMMUNOLOGY AND ALLERGY CLINICS OF NORTH AMERICA, V. 23, N. 1, P. 15-39, 2// 2003. ISSN 0889-8561. DISPONÍVEL EM: < HTTP:/WWW.SCIENCEDIRECT.COM/SCIENCE/ARTICLE/PII/S0889856102000565 $>$. 
8. ANEXOS

I. COMPROVANTE DE APROVAÇÃO EM COMITÊ DE ÉTICA

\begin{tabular}{c} 
UNIVERSIDADE DE BRASILIA \\
FACULDADE DE MEDICINA \\
Comitê de Ética em Pesquisa em Seres Humanos \\
\hline
\end{tabular}

ANÁLISE DE PROJETO DE PESQUISA

Registro de Projeto: CEP-FM 061/2011.

Titulo: "Associação de marcadores imunológicos ê genomicos com doença aterosclerotica no paciente ambulatorial idoso".

Pesquisador Responsável: Otávio de Tolèdo Nobrega

Documentos analisados: Folha de rosto. carta de encaminhamento. declaração de responsabilidade. protocolo de pesquisa. termo de consentimento livre e esclarecido, cronograma. bibliografia pertinente e currículo (s) de pesquisador (es).

Data de entreoa: 22/08/2011.

Parecer do (a) relator (a)

(X) Aprovação

( ) Não aprovação.

Data da primeira análise pelo CEP-FM/UNB: 10/10/2011.

Data do parecer final do projeto pelo CEP-FM/UNB: 26/12/2011.

PARECER

Com base na Resolução CNS/MS n 196/96 e resoluções posteriores. que regulamentam a matéria. a coordenação do Comitê de Ética em Pesquisa da Faculdade de Medicina da Universidade de Brasilia decidiu APROVAR "ad referendum" do CIP-F.M. conforme parecer do (a) relator (a). o projeto de pesquisa acima especificado quanto aos seus aspectos éticos.

1. Modificações no protocolo devem ser submetidas ao CEP. assim como a notilicaçăo imediata de eventos adversos graves

2. $\mathrm{O}(\mathrm{s})$ pesquisador (es) deve ( $\mathrm{m}$ ) apresentar relatórios periódicos do andamento da pesquisa ao CEPFM, sendo o $1^{\circ}$ previsto para 20 de julho de 2012.

Brasilia. 18 de Janciro de 2012

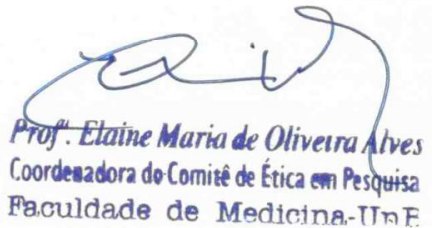




\title{
II. TERMO DE CONSENTIMENTO LIVRE E ESCLARECIDO
}

\author{
UNIVERSIDADE DE BRASÍLIA ñ UNB \\ UNIVERSIDADE CATÓLICA DE BRASÍLIA ñ UCB \\ INSTITUTO DE CARDIOLOGIA - BIOCÁRDIOS
}

óAssociação de marcadores imunológicos e genômicos com doença aterosclerótica no paciente ambulatorial idoso.

O envelhecimento pode ocasionar doenças ou o agravamento de suas manifestações no organismo, diminuindo a qualidade de vida da pessoa. Por isso, nós do Instituto de Cardiologia Biocárdios no Distrito Federal e do Hospital da Universidade Católica de Brasília (HUCB), em conjunto com pesquisadores da Universidade de Brasília (UnB), estamos trabalhando na busca por sinais clínicos e de laboratório que permitam antever o desenvolvimento de doenças associadas ao envelhecimento, sobretudo aquelas doenças relacionadas a problemas em importantes artérias do corpo humano, a exemplo da artéria coronariana e das artérias carotídeas.

Assim sendo, com o objetivo de encontrar características do organismo que possam contribuir para o diagnóstico precoce de distúrbios nestas artérias, necessitamos do seu consentimento para realizamos uma avaliação médica e por exames laboratoriais, incluindo avaliações por ultrasonografia, tomografia computadorizada, exames de sangue e testes genéticos. Esta pesquisa poderá possibilitar uma melhoria da compreensão do processo de envelhecimento do ser humano, o que por sua vez permitirá melhorar o atendimento e o aconselhamento prestados aos idosos assistidos tantos pelos programas públicos e quantos privados de saúde.

Dependendo dos resultados e da avaliação, o(a) senhor(a) poderá ser incluído no grupo de portadores ou não-portadores de uma doença ou achado clínico importante sobre as artérias estudadas.

Termo de consentimento livre e esclarecido

Fui informado que o protocolo experimental consistirá basicamente em uma avaliação médica e por exames laboratoriais e por imagem, e que tais procedimentos não comprometerão minhas atividades cotidianas e não serão diferentes daqueles procedimentos rotineiramente recomendados pela prática clínica moderna. Durante as consultas, responderei perguntas sobre os medicamentos que utilizo, como sigo o(s) tratamento(s) recomendado(s), do que me alimento e em quais quantidades, mas terei plena liberdade de me recusar a responder caso eu não queira. Fui informado(a) ainda que este trabalho não oferecerá riscos expressivos à minha saúde, já que não realizarei movimentos anormais, não terei mudança de rotina, não entrarei em contato com quaisquer substâncias nocivas, nem terei qualquer instrumento introduzido em meu corpo, a não ser por ocasião de uma única coleta de um pequeno volume (15 mililitros) de sangue, onde me será assegurada utilização de agulhas descartáveis. A equipe do projeto se responsabilizou por prestar esclarecimentos a mim a qualquer momento da pesquisa, inclusive relativos a exames de laboratório realizados, disponibilizando para tanto as formas de contato presentes no rodapé desta página.

O pesquisador garantiu sigilo sobre minha identidade, ficando os dados sob sua guarda, não sendo permitido acesso por pessoas não relacionadas à pesquisa. O pesquisador responsabilizou-se por qualquer dano que eu venha a sofrer, e também explicou que as amostras coletadas poderão ser estocadas para outras pesquisas, mas que serei contatado para conceder minha autorização para cada nova utilização.

Assim, por este documento, dou meu consentimento à exploração dos dados coletados por este projeto de pesquisa.

Brasília, de de 20

Nome do paciente

Nome do profissional que prestou informações 
III. FICHA DE AVALIAÇÃO CLINICA

\section{HOSPITAL DA UNIVERSIDADE CATOLICA DE BRASILIA - HUCB Curso de Medicina - Disciplina de Geriatria/Gerontologia}

FICHA CLINICA/ANAMNESE

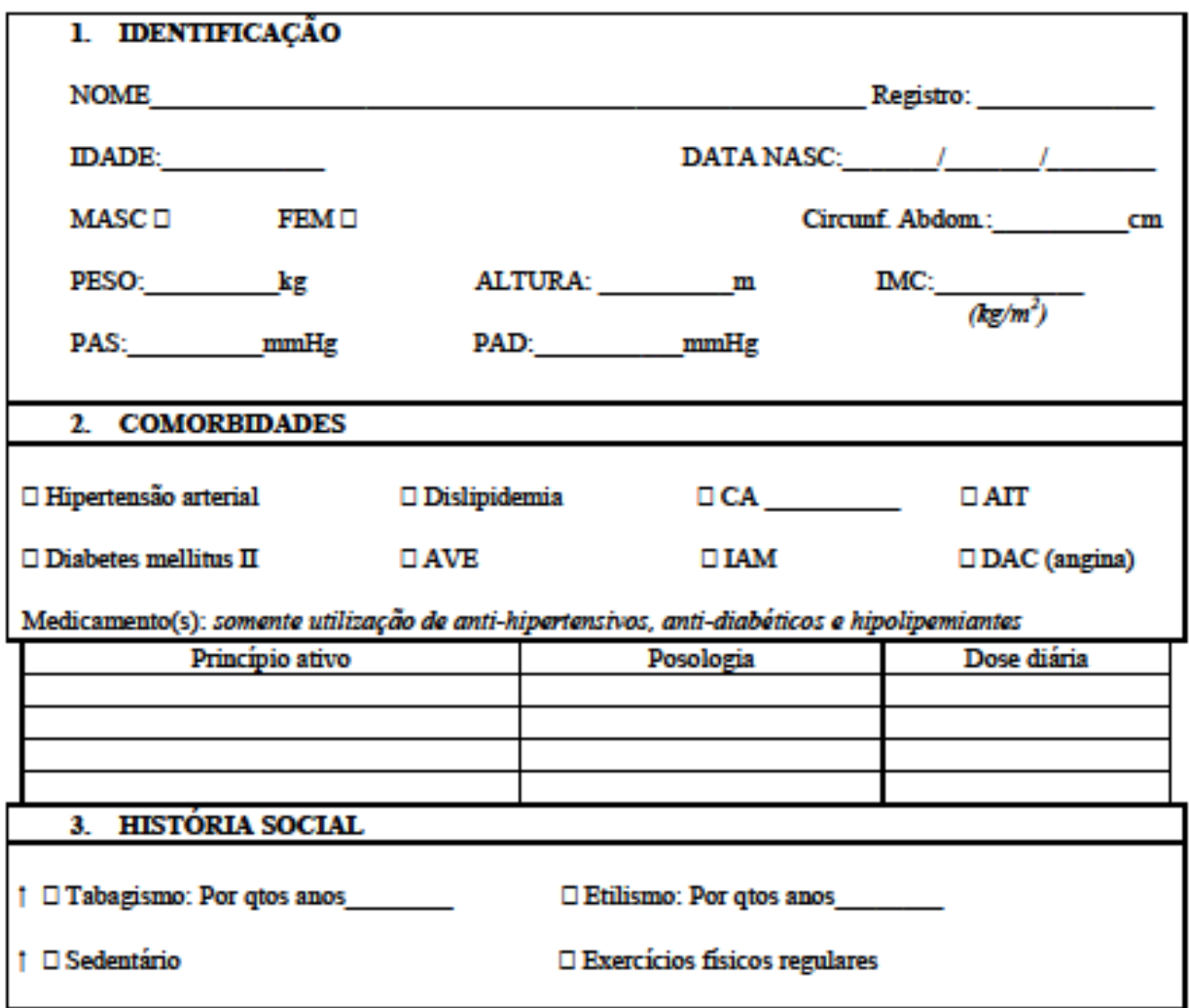

\begin{tabular}{|lll|}
\hline EXAMES LABORATORIAIS: & \\
\hline TSH: & HEMOGLOBINA: & \\
\hline PCR US: & HOMOCISTEINA: & \\
\hline CREATININA: & COCKCROFT-GAULT: & \\
\hline COL. TOTAL: & TRIGLIC: & \\
\hline HDL: & LDL: & \\
\hline VLDL: & GAMA GT: & TGO: \\
\hline GLICEMIA: & INSULINEMIA: & \\
\hline HbAlC: & HOMA: & \\
\hline ECG: & & \\
\hline & & \\
\hline
\end{tabular}

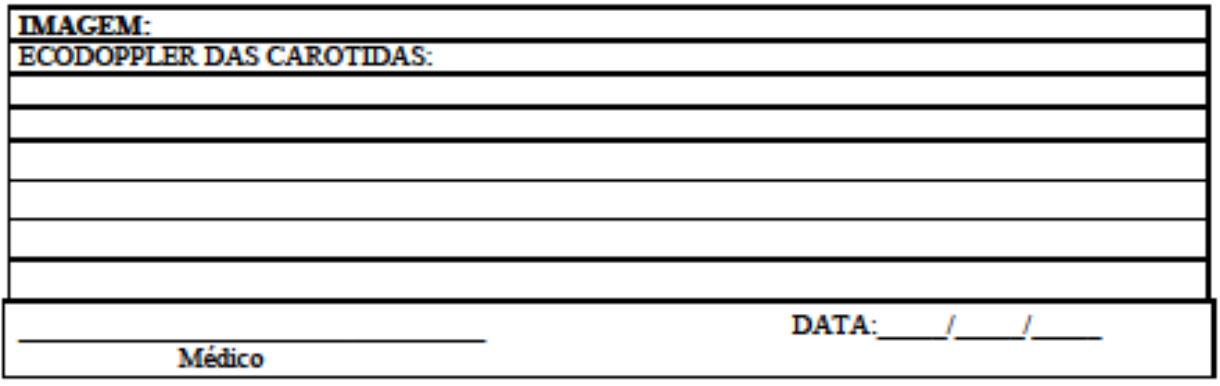

Prof. Clayton Franco Moraes - claytonfaucb.br - Prof "Lucy Gomes - luacygomes/apos.ucb.br HUCB - QS 05, lote 22 - Avenida Areal Águas Claras/DF CEP: 71.955-000 - TELEFONE: 61-3451-1000 - wwwwhuab.apm.br 


\section{Otávio T. Nóbrega}

Assunto:

ENC- 6131491011335223 Circulating inflammatory mediators independently associate with atherosclerosis in the left (not right) carotid territory of older subjects

De: Immunity \& Ageing Editorial [mailtosimmunity ageing®unipa.it]

Enviada em: sabado, 23 de maio de 2015 18:56

Para: Dr Otavio T. Nobrega

Assunto: 6131491011335223 Circulating inflammatory mediators independently associate with atherosclerosis in the left (not right) carotid territory of older subjects

Article title: Circulating inflammatory mediators independently associate with atherosclerosis in the left (not right) carotid territory of older subjects

MS ID : 6131491011335223

Authors : Wilcelly Machado-Silva, Adriane Dallanora Henriques, Gleim Dias Souza, Lucy Gomes, Aparecido

Pimentel Ferreira, Ciro José Brito, Cláudio Córdova, Otávio Toledo Nóbrega

Journal : Immunity \& Ageing

Dear Dr Nobrega

Thank you for submitting your article. This acknowledgement and any queries below are for the contact author. This e-mall has also been copied to each author on the paper, as well as the person submitting. Please bear in mind that all queries regarding the paper should be made through the contact author.

A pdf file has been generated from your submitted manuscript and figures. We would be most grateful if you could check this file and let us know if any aspect is missing or incorrect. Any additional files you uploaded will also be sent in their original format for review.

httpc//www.immunityageing.com/imedia/6131491011335223_article.pdf (BOK)

For your records, please find below lirk(s) to the correspondence you uploaded with this submission. Please note there may be a short delay in creating this file.

httpc//www.immunityageing.com/imedia/1890159288133522_comment.pdf

Your manuscript will be considered by our editors and will aim to contact you with an initial decision on the manuscript within .

In the meantime, if you have any queries about the manuscript you may contact us on immunity_ageingeunipa.it. We would also welcome feedback about the online submission process, which can be sent to info@biomedcentral.com.

Best wishes,

The Immunity \& Ageing Editorial Team

e-mail: immunity_ageingeunipa.it

Web: http://www.immunityageing.com/ 
Circulating inflammatory mediators independently associate with atherosclerosis in the left (not right) carotid territory of older subjects

Type: Research paper

Running title: cytokines and carotid intima-media thickness

\section{Authors:}

Wilcelly Machado-Silva ${ }^{1}$, Adriane Dallanora Henriques ${ }^{1}$, Gleim Dias Souza ${ }^{2}$, Lucy Gomes ${ }^{2}$, Aparecido Pimentel Ferreira ${ }^{3}$, Ciro José Brito ${ }^{4}$, Cláudio Córdova ${ }^{2}$, Clayton Franco Moraes ${ }^{2}$, Otávio Toledo Nóbrega ${ }^{1, *}$

\section{Affiliations:}

${ }^{1}$ Universidade de Brasília (UnB), Brasília - DF, Brazil.

${ }^{2}$ Universidade Católica de Brasília (UCB-DF), Taguatinga - DF, Brazil.

${ }^{3}$ Núcleo Interdisciplinar de Pesquisa, Faculdades Promove, Brasília, DF, Brazil

${ }^{4}$ Universidade Federal de Sergipe, Sergipe, Brazil.

* Corresponding author at: Universidade de Brasília (UnB), Campus Universitário Darcy Ribeiro, 70.910-900, Brasília - DF, Brazil. Phone: + 556131071913.

E-mail adresses: wilcellymachado@gmail.com (W. Machado-Silva), adrianedallanora@gmail.com (A.D. Henriques), gleimdias@grupodigimed.com.br (G.D. Souza), lucygomes2006@hotmail.com (L. Gomes), cirojbrito@gmail.com (C.J. Brito), cordova@ucb.br (C. Córdova), cidopimentel@yahoo.com.br (A.P. Ferreira), otavionobrega@unb.br(O.T. Nóbrega). 


\section{ABSTRACT}

BACKGROUND: Atherosclerosis is a chronic process triggered by endogenously modified structures within arterial walls, particularly oxidized lipoproteins, that may shaped by innate and adaptive immune mediators. There is evidence that poor architecture of the carotid arteries, expressed as augmented intima-media thickness (cIMT), is a strong predictor of future cerebrovascular events and correlates with a systemic inflammatory milieu.

OBJECTIVE: to investigate the relationship of a profile of blood-carried inflammatory mediators with measures of cIMT in elderly subjects, taking traditional risk factors into account.

METHODS: Clinical inspection for present and past chronic conditions and events as well as biochemical and anthropometric measurements were performed for patients aged 60 years or older in ambulatory care. Scores of cIMT were obtained bilaterally in the distal common carotid artery wall. Serum concentrations of ten different cytokines were assessed by beadbased, multiplexed flow cytometry immunoassays.

RESULTS: Correlation analysis between log-transformed cytokines levels and cIMT scores demonstrated significant association of IL16, IL6, IL8, IL10 and TNFŬ(P Ò0.005) with left (but not right) cIMT scores. Stepwise multivariate regression showed that TNFŬ IL16 and IL6 levels accounted for $29.5 \%\left(\mathrm{R}^{2}=0.209\right)$ of the variance in scores of the cIMT scores. Comparison of cytokines levels across increasing tertiles of left cIMT reproduced a positive association with TNFŬand IL16 levels.

CONCLUSION: Five out of ten immune mediators independently correlated with cIMT of older subjects in a territory-sensitive manner. This possible contribution of immune mediators to an atherosclerotic process probably relates to the inflammaging phenomenon.

Keywords: Atherosclerosis, carotid artery, intima-media thickness, cytokines, inflammation, aging. 


\section{Introduction}

Atherosclerosis results from a chronic inflammatory process within the arterial wall that is initiated mainly in response to endogenously modified structures, particularly oxidized lipoproteins. It is widely recognized that stimulation by circulating mediators of the innate and adaptive immune responses leads to further alteration of the vascular wall, promoting disease progression and complications ${ }^{1 ; 2}$, with evidence that imbalance between pro- and anti-inflammatory factors play a critical role in early vascular endothelial dysfunction ${ }^{3 ; 4}$.

Atherosclerosis affects large and medium sized arteries, of both elastic and muscular natures. The major concern is the possibility of causing thrombosis or severe stenosis in the arteries, leading to adverse, life-threatening events as myocardial infarction, ischemic stroke, ischemic damage to the kidneys and intestines, and other clinical manifestations ${ }^{5}$. Avoiding death and morbidity from circulatory causes justifies a great deal of interest in identifying asymptomatic high-risk patients ${ }^{6}$. In this regard, imaging comprises a safe and affordable noninvasive tool for providing valuable information on peripheral circulatory beds, such as the carotid arteries in which identification of subclinical atherosclerosis may improve risk stratification for the development of cerebrovascular events ${ }^{7 ; 8}$. Taking evidence that an increase in carotid intima-media thickness (IMT) in the order of $0.1 \mathrm{~mm}$ provides per se a relevant contribution to the onset of stroke (hazard ratio $=1.17$ ) ${ }^{9}$, current literature often reiterates that features of the carotid morphology stand as strong predictors of future vascular events $9 ; 10 ; 11 ; 12$.

In this scenario, the aim of the study was to investigate the relationship between a panel of circulating inflammatory markers assessed by a high-throughput method with common clinical and biochemical traits of vascular relevance as well as with image assessments of the carotid arteries. 


\section{Methods}

Sample

A sample of consecutive non-institutionalized patients aged 60 years or older, without clinical symptoms of severe atherosclerotic, was recruited between 2011 and 2013 at two general geriatrics outpatient clinics located at the metropolitan area of the Federal District, Brazil, to assess determinants of circulatory disorders as the Prognosis and Therapeutics in Geriatrics cohort. The clinics are the Geriatrics Service of the Hospital of the Universidade Católica de Brasília (HUCB) and an ambulatory at the Hospital of the Universidade de Brasília (UnB), which accept patients with similar sociodemographic characteristics. Patients recruited from UnB were all directed to the HUCB for the purposes of baseline evaluation and follow up, for the sake of developing clinical protocols and providing homogeneous care by one same staff. Inclusion criteria were to spontaneously seek primary or secondary care for circulatory events. Exclusion criteria to integrate these analyses were: (i) autoimmune disease, (ii) neoplasms of any type, current or past, (iii) obstructive pulmonary disease severe symptomatic or asymptomatic, (iv) severe chronic kidney disease (clearance of creatinine than $25 \mathrm{ml} / \mathrm{min} / 1.73 \mathrm{~m}^{2}$ ), (v) chronic or recurrent infections, (vi) liver disease defined by changes in biochemical and/or liver function tests or imaging studies consistent with severe liver disease, (vii) failure heart (ejection fraction <50\%). There was no active search for patients with any particular condition.

The project was approved by the Research Ethics Committee of the Medical Faculty of the University of Brasilia, under the Office in 061/2011. All participants signed a free and informed consent.

Clinical and biochemical assessments 
Clinical inspection of each patient consisted in assessing presence of diabetes and hypertension, current use of drugs for chronic conditions, previous circulatory events as myocardial or cerebrovascular infarction and familial history of major circulatory diseases as well as lifestyle aspects as sedentary and smoking habits. Diagnosis of type 2 diabetes included self-reported of the condition and/or fasting glycosylated hemoglobin (Hb1 Ac) Ó $6.5 \% 35$. Practitioners of physical exercises were those exhibiting 30 minutes or over of exercises at any bout for at least four days a week ${ }^{36}$, while the smoking habit was defined as consumption of $>100$ cigarettes over a lifetime ${ }^{37}$. For metabolic analysis, the following measures were obtained according to routine clinical biochemistry and expressed in standard units: blood glucose, glycated hemoglobin (HbA1c), insulin, total cholesterol and fractions, triglycerides, C-reactive protein high sensitivity, HOMA index, TSH and homocysteine. Anthropometric analyses assessed body mass index (BMI, in $\mathrm{kg} / \mathrm{m}^{2}$ ) and waist circumference $(\mathrm{cm})$.

\section{Measurement of intima-media thickness}

The carotid intima-media thickness (cIMT) was studied noninvasively in all participants by means of high-resolution ultrasound using Philips equipment (IE 33 model) with a linear transducer 3-9 MHz.

The cIMT measurements were performed bilaterally in the distal common carotid artery wall of the bulb and at the origin of the internal carotid artery, through an automatized edge detection program which is capable of showing the maximum mean value of 150 measurements of any $10 \mathrm{~mm}$ segment. The maximum IMT was measured manually as the mean of 3 independent measurements. All clinical procedures and imaging evaluations were performed at HUCB. 


\section{Inflammatory panel}

On the occasion of the biochemical evaluations, whole blood was drawn and the serum obtained was kept frozen at $-80{ }^{\circ} \mathrm{C}$ until thawed for the assessment of the immune mediators. Cytokine concentrations were assessed by a multiplexed flow cytometry method using two sets of bead-based immunoassays known as the Human Th1/Th2/TH17 kit and the Human Inflammatory kit manufactured by BD Biosciences ${ }^{\circledR}$ (San Diego, CA, USA), used according to the manufacturer protocols and which all together yielded measurements for ten different circulating mediators, as follows: IL16, IL2, IL4, IL6, IL8, IL10, IL12.p70, IL17A, INF3 and TNFŬ.

Briefly, the lyophilized cytokine standards and the serum samples were processed and the results acquired using the BD FACSCalibur flow cytometer, FL4 channel. Three hundred events were acquired for each cytokine bead used. Data were analyzed using the FCAP software, version 3.0 (BD Biosciences ${ }^{\circledR}$, San Diego, CA, USA). Standard curves for each cytokine were generated using standard mixture of mediators supplied. The concentration in each serum was determined by interpolation from the corresponding standard curve.

\section{Statistical analyses}

To address the aim of evaluating the occurrence and strength of the association between circulating levels of immune mediators and scores of intima-media thickness, our statistical analyses started at obtaining correlation coefficients between these traits and other (continuous and categorical) anthropometric, clinical and biochemical variables of potential confounding effect in the main model. For that, normal distribution of all variable was assessed using the Kolmogorov-Smirnov test and, whenever necessary and possible, logarithmic transformations were applied to achieve a closer-to-normal distribution. 
The association between continuous variables was evaluated using the Pearsonôs correlation test, whereas the involvement of a least one categorical variable in the model was dealt using the Spearmanôs counterpart. For these latter analyses, presence or absence of a feature was represented by 1 or 0 , respectively. Whenever an interaction was noticed, partial correlation analyses were run using adjustment for the confounding variable(s) or condition(s). For these correlation analyses, a P-value (two-tailed) was rendered significant following the Bonferroniôs principle of correction for multiple comparisons whenever a given trait is tested across $n$ independent variables $(n=10$ tests; $\therefore \breve{U}=0.005)$.

Also, analysis of variance was used to test whether log-transformed cytokine concentrations varied across tertiles of IMT scores in the sample, with adjustment to covariates and P Ò 0.05 as threshold of significant. Finally, linear multivariate regression analysis, stepwise method, was performed to assess at which extent serum concentrations of the inflammatory mediators explain the variability of the carotid IMT scores.

All analyses were performed with the Statistical Package for the Social Sciences (SPSS) for Windows (version 17.0).

\section{Results}

Data from 168 patients admitted between 2011 and 2013 were analyzed for clinical and inflammatory profile. Table 1 shows anthropometric, clinical and metabolic features. The overall profile of the patients is compatible with borderline metabolic disorders, with a high prevalence of hypertensive, diabetic and sedentary cases. On top of that, previous cardiovascular events were not rare in the sample, as illustrated by cases of prior stroke (9.5\%), acute myocardial infarction (4.2\%) and coronary artery disease (1.8\%).

At first, correlation analysis of raw levels of serum cytokines and of right and left carotid IMT scores across categorical clinical and healthcare features showed no association 
of either immune mediators or artery architecture with any previous condition of risk to the onset of CVDs or with aspects that modulate/treat such conditions $(\mathrm{P}>0.005)$ (Table 2). The correlation analysis of logarithmically(log)-transformed levels of the panel of cytokines and IMT scores across continuous clinical and biochemical features has demonstrated correlation and significance level of HDL-c with $\log _{10} \mathrm{IL16}(\mathrm{P}<0.001)$ as well as with $\log _{10} \mathrm{IL6}(\mathrm{P}<$ 0.004). However, none of these latter features correlated with the raw right and left carotid IMT (Table 3).

In the correlation analysis between log-transformed levels of circulating cytokines and raw carotid IMT scores, the immune mediators IL16, IL6, IL8, IL10 and TNFŬdemonstrated a significant level of positive association (P Ò 0.005) with left but not right IMT scores, whereas the other log-transformed mediators (IFNכ, IL2, IL4, IL12p70 and IL17a) showed not to associate with either cIMT territories (Table 4). Accordingly, comparison of the logtransformed circulating levels of the cytokines across the individuals grouped in increasing tertiles of intima-media thickness of the left carotid artery confirmed a positive correlation with levels of TNFǓ and IL16, with adjustment to HDL-c (Figure 1), whereas means of IL6, IL8 and IL10 did not reach significant difference across tertiles with this approach. In line, stepwise multivariate regression confirmed that $\log _{10}$ TNFŬ was the first most predictive variable in the model $\left(\mathrm{R}^{2}=0.209\right)$, which added to $\log _{10} \mathrm{IL} 1 \mathrm{~b}$ and $\log _{10} \mathrm{IL6}$ in this order accounted for $29.5 \%$ of the variance in scores of intima-media thickness, independently of other risk factors for atherosclerosis.

\section{Discussion}

We seek in this paper to investigate the relationship of clinical/biochemical features, healthcare pratices and inflammatory traits with an important aspect of the carotid morphology. Mainly, we found that log-transformed levels of IL16, IL6, IL8, IL10 and TNFŬ, 
independently correlated carotid IMT scores in its left branch. Our results corroborate the involvement of a set of circulating pro-inflammatory cytokines in the arteriosclerotic process of this cerebrovascular milieu. Interestingly, no other clinical/biochemical or healthcare variables assessed herein correlated with the carotid morphology, apart from an expected trend towards a positive association of the cIMT scores with prior events of stroke.

Our findings concur with epidemiological findings where patients with chronic inflammatory diseases, such as rheumatoid arthritis (RA), bear a significantly greater risk of developing coronary artery disease (CAD) than the general population ${ }^{13}$, along with greater thickness of the carotid walls ${ }^{14}$. Also, the fact that immune mediators as TNFŬand IL6 (this latter as an usual outcome of TNFŬ production) are significantly associated with premature atherosclerosis in the carotid artery ${ }^{15 ;}{ }^{16}$, even independently of traditional circulatory risk factors $17 ; 18$, is recurrent in the literature. In line, use of TNFŬ antagonist improved endothelial function and reduced IMT in the carotid bed of RA patients ${ }^{19 ; 20 ; 21}$. Evidence that IL16 plays an important role in atherosclerosis is also available. Being synthesized by endothelial cells, vascular smooth muscle cells (VSCMs) and macrophages, it can induce cytokine production, activation of endothelial cells, increased vascular permeability and proliferation of VSMCs ${ }^{22 ;} 23$. Lack of IL16 in ApoE-deficient mice reduces atherosclerotic lesions whereas its increased expressions trigger vascular cell adhesion molecule-1 and monocyte chemotactic protein-1 in the aorta endothelium, possibly increasing recruitment of monocytes/macrophages to the intima ${ }^{24}$.

In this set of cytokines positively correlated with carotid IMT, IL10 is the only that presents anti-inflammatory properties. As an important player in the immune response down regulation, IL10 production is known to reduce the extension and/or severity of atherosclerosis, and stabilize vulnerable phenotypes ${ }^{25 ;} 26$. Expression of IL10 induced by oxidized LDL decreases the release of proinflammatory cytokines so to prevent the excessive 
innate or acquired immune activation (and due fibrotic outcomes) within the layers of elastic or muscular of arteries ${ }^{27 ;} 28$. Therefore, the augmented levels of IL10 among carriers of greater cIMT scores may simply be the result of a compensatory mechanism in response to the systemic elevation in proinflammatory signaling.

The left common carotid artery (CCA) has already proven to be more vulnerable for atherosclerosis, usually with larger plates and stiffness ${ }^{29 ; 30}$, than its right counterpart. The anatomy of the circulatory system may at least in part explain the pathological asymmetry between branches. The left CCA comes directly from the arch of the aorta whereas the right CCA from the innominate artery ${ }^{31}$. This anatomical difference exposes the left CCA to greater pressure (and due higher sheer stress) levels from the aortic arch, since the right CCA is subjected to lower blood flow from the ascending aorta ${ }^{30}$. In this scenario, it is reasonable to assume that vascular lesions triggered by greater hydrostatic pressure can be enhanced proportionally to the pro-inflammatory milieu, which might not be an important pathophysiological modulator of lesions at less turbulent sites.

All in all, our study is in line with robust evidence that the onset of age-related chronic conditions are accompanied by a subclinical, systemic state of low-grade inflammation, named inflammaging. To the authors, unveiling pro-inflammatory mediators (IL16, IL6, IL8 and TNFŬ) as possible contributors to the atherosclerosis-prone phenotype investigated herein can be an exemplification of this phenomenon ${ }^{32 ; 33}$. But our study has limitations, despite all methodological care. The cross-sectional design prevents determining causality among variables. Moreover, number and morphological profile on atheroma plaques was beyond our assessment capability. Also, the possible contribution of nutrition and other life style variables was not considered herein.

In conclusion, we show that blood-borne levels of five out of 10 immune mediators independently correlated with scores of left (but no right) carotid intima media thickness 
among older subjects, with stronger associations obtained with TNFŬ, IL16 and IL6, in this order. Our work suggests that this possible contribution of immune mediators to the atherosclerotic process in the vascular territory investigated probably relates to the inflammaging phenomenon.

Acknowledgments: The research was supported with grants \#471016/2011-0 (CNPq) and \#193.000.032-2012 (FAPDF), with a stipend to W. Machado-Silva (CAPES) and a fellowship for productivity in research to O.T. Nóbrega (CNPq).

Conflict of interest: None.

\section{Author contributions:}

W Machado-Silva and AD Henriques: assessed the circulating immune mediators studied.

GD Souza: performed the carotid imaging evaluations of the study

L Gomes and CF Moraes: executed the medical component of the study.

CJ Brito, AP Ferreira and C Córdova: substantially advised in the interpretation of results.

OT Nóbrega and CF Moraes: designed and coordinated the study.

W Machado-Silva and OT Nóbrega: analysed and interpreted the results; participated in the preparation of the manuscript. 


\section{References}

$1 \quad$ MALLAT, Z. et al. The role of adaptive T cell immunity in atherosclerosis. J Lipid Res, v. 50 Suppl, p. S364-9, Apr 2009. ISSN 0022-2275 (Print)

0022-2275 (Linking). Disponível em: < http://www.ncbi.nlm.nih.gov/pubmed/19050311 >.

2 MORRIS, P. B. et al. Review of Clinical Practice Guidelines for the Management of LDLRelated Risk. Journal of the American College of Cardiology, v. 64, n. 2, p. 196-206, 7/15/ 2014. ISSN 0735-1097. Disponível em: < http://www.sciencedirect.com/science/article/pii/S0735109714025911 >.

3 HWANG, M. H.; KIM, S. Type 2 Diabetes: Endothelial dysfunction and Exercise. J Exerc Nutrition Biochem, v. 18, n. 3, p. 239-47, Sep 2014. ISSN 2233-6834 (Print)

2233-6834 (Linking). Disponível em:<http://www.ncbi.nlm.nih.gov/pubmed/25566460 >.

4 BERLINER, J. A. et al. Atherosclerosis: basic mechanisms. Oxidation, inflammation, and genetics. Circulation, v. 91, n. 9, p. 2488-96, May 1 1995. ISSN 0009-7322 (Print)

0009-7322 (Linking). Disponível em: < http://www.ncbi.nlm.nih.gov/pubmed/7729036 >.

5 LIBBY, P.; RIDKER, P. M.; HANSSON, G. K. Progress and challenges in translating the biology of atherosclerosis. Nature, v. 473, n. 7347, p. 317-25, May 19 2011. ISSN 1476-4687 (Electronic)

0028-0836 (Linking). Disponível em: < http://www.ncbi.nlm.nih.gov/pubmed/21593864 >.

6 STEIN, J. H. et al. Use of carotid ultrasound to identify subclinical vascular disease and evaluate cardiovascular disease risk: a consensus statement from the American Society of Echocardiography Carotid Intima-Media Thickness Task Force. Endorsed by the Society for Vascular Medicine. J Am Soc Echocardiogr, v. 21, n. 2, p. 93-111; quiz 189-90, Feb 2008. ISSN 1097-6795 (Electronic)

0894-7317 (Linking). Disponível em: < http://www.ncbi.nlm.nih.gov/pubmed/18261694 >.

7 PETERS, S. A. et al. Improvements in risk stratification for the occurrence of cardiovascular disease by imaging subclinical atherosclerosis: a systematic review. Heart, v. 98, n. 3, p. 17784, Feb 2012. ISSN 1468-201X (Electronic)

1355-6037 (Linking). Disponível em: < http://www.ncbi.nlm.nih.gov/pubmed/22095617 >.

8 VAN DEN OORD, S. C. et al. Carotid intima-media thickness for cardiovascular risk assessment: systematic review and meta-analysis. Atherosclerosis, v. 228, n. 1, p. 1-11, May 2013. ISSN 1879-1484 (Electronic)

0021-9150 (Linking). Disponível em: < http://www.ncbi.nlm.nih.gov/pubmed/23395523 >. 
LORENZ, M. W. et al. Prediction of clinical cardiovascular events with carotid intima-media thickness: a systematic review and meta-analysis. Circulation, v. 115, n. 4, p. 459-67, Jan 30 2007. ISSN 1524-4539 (Electronic)

0009-7322 (Linking). Disponível em: < http://www.ncbi.nlm.nih.gov/pubmed/17242284 >.

YEBOAH, J. et al. Mediation of cardiovascular risk factor effects through subclinical vascular disease: the Multi-Ethnic Study of Atherosclerosis. Arterioscler Thromb Vasc Biol, v. 34, n. 8, p. 1778-83, Aug 2014. ISSN 1524-4636 (Electronic)

1079-5642 (Linking). Disponível em: < http://www.ncbi.nlm.nih.gov/pubmed/24876350 >. systematic review and meta-analysis. Int J Neurosci, Aug 28 2014. ISSN 1563-5279 (Electronic)

0020-7454 (Linking).

DARABIAN, S. et al. The role of carotid intimal thickness testing and risk prediction in the development of coronary atherosclerosis. Curr Atheroscler Rep, v. 15, n. 3, p. 306, Mar 2013. ISSN 1534-6242 (Electronic)

1523-3804 (Linking). Disponível em: < http://www.ncbi.nlm.nih.gov/pubmed/23328906 >.

WILSON, P. W. Evidence of systemic inflammation and estimation of coronary artery disease risk: a population perspective. Am J Med, v. 121, n. 10 Suppl 1, p. S15-20, Oct 2008. ISSN 1555-7162 (Electronic)

0002-9343 (Linking). Disponível em: < http://www.ncbi.nlm.nih.gov/pubmed/18926165 >.

VAN SIJL, A. M. et al. Carotid intima media thickness in rheumatoid arthritis as compared to control subjects: a meta-analysis. Semin Arthritis Rheum, v. 40, n. 5, p. 389-97, Apr 2011. ISSN 1532-866X (Electronic)

0049-0172 (Linking). Disponível em: < http://www.ncbi.nlm.nih.gov/pubmed/20889191 >.

OKAZAKI, S. et al. Association of interleukin-6 with the progression of carotid atherosclerosis: a 9-year follow-up study. Stroke, v. 45, n. 10, p. 2924-9, Oct 2014. ISSN 15244628 (Electronic)

0039-2499 (Linking). Disponível em: < http://www.ncbi.nlm.nih.gov/pubmed/25139874 > healthy middle-aged men. Eur Heart J, v. 23, n. 5, p. 376-83, Mar 2002. ISSN 0195-668X (Print). em: http://www.ncbi.nlm.nih.gov/entrez/query.fcgi?cmd=Retrieve\&db=PubMed\&dopt=Citation \&list uids $=11846495>$. 
RHO, Y. H. et al. Inflammatory mediators and premature coronary atherosclerosis in rheumatoid arthritis. Arthritis Rheum, v. 61, n. 11, p. 1580-5, Nov 15 2009. ISSN 0004-3591 (Print)

0004-3591 (Linking). Disponível em: < http://www.ncbi.nlm.nih.gov/pubmed/19877084 >. disease: a collaborative meta-analysis of 82 studies. Lancet, v. 379, n. 9822, p. 1205-13, Mar 31 2012. ISSN 1474-547X (Electronic)

0140-6736 (Linking). Disponível em: < http://www.ncbi.nlm.nih.gov/pubmed/22421339 >. the QUEST-RA study. Arthritis Res Ther, v. 10, n. 2, p. R30, 2008. ISSN 1478-6362 (Electronic)

1478-6354 (Linking). Disponível em: < http://www.ncbi.nlm.nih.gov/pubmed/18325087 >. function in patients with rheumatoid arthritis. Circulation, v. 106, n. 17, p. 2184-7, Oct 22 2002. ISSN 1524-4539 (Electronic)

0009-7322 (Linking). Disponível em:< http://www.ncbi.nlm.nih.gov/pubmed/12390945 >.

DEL PORTO, F. et al. Response to anti-tumour necrosis factor alpha blockade is associated with reduction of carotid intima-media thickness in patients with active rheumatoid arthritis. Rheumatology (Oxford), v. 46, n. 7, p. 1111-5, Jul 2007. ISSN 1462-0324 (Print)

1462-0324 (Linking). Disponível em: < http://www.ncbi.nlm.nih.gov/pubmed/17449484 >.

FEARON, W. F.; FEARON, D. T. Inflammation and cardiovascular disease: role of the interleukin-1 receptor antagonist. Circulation, v. 117, n. 20, p. 2577-9, May 20 2008. ISSN 1524-4539 (Electronic)

0009-7322 (Linking). Disponível em: < http://www.ncbi.nlm.nih.gov/pubmed/18490534 >. disease. Arterioscler Thromb Vasc Biol, v. 16, n. 8, p. 1000-6, Aug 1996. ISSN 1079-5642 (Print)

1079-5642 (Linking). Disponível em:< http://www.ncbi.nlm.nih.gov/pubmed/8696938 >. deficient mice. Arterioscler Thromb Vasc Biol, v. 23, n. 4, p. 656-60, Apr 1 2003. ISSN 15244636 (Electronic)

1079-5642 (Linking). Disponível em: < http://www.ncbi.nlm.nih.gov/pubmed/12615675 > 
TZIAKAS, D. N. et al. Anti-inflammatory cytokine profile in acute coronary syndromes: behavior of interleukin-10 in association with serum metalloproteinases and proinflammatory cytokines. Int J Cardiol, v. 92, n. 2-3, p. 169-75, Dec 2003. ISSN 0167-5273 (Print)

0167-5273 (Linking). Disponível em: < http://www.ncbi.nlm.nih.gov/pubmed/14659849 >.

MORAITIS, A. G. et al. Elevated interleukin-10: A new cause of dyslipidemia leading to severe HDL deficiency. J Clin Lipidol, v. 9, n. 1, p. 81-90, Jan-Feb 2015. ISSN 1933-2874 (Print)

1876-4789 (Linking). Disponível em: < http://www.ncbi.nlm.nih.gov/pubmed/25670364 >.

BENITEZ, S. et al. Pro-inflammatory action of LDL(-) on mononuclear cells is counteracted by increased IL10 production. Biochim Biophys Acta, v. 1771, n. 5, p. 613-22, May 2007. ISSN 0006-3002 (Print)

0006-3002 (Linking). Disponível em: < http://www.ncbi.nlm.nih.gov/pubmed/17442617 >

POTTEAUX, S. et al. Leukocyte-derived interleukin 10 is required for protection against atherosclerosis in low-density lipoprotein receptor knockout mice. Arterioscler Thromb Vasc Biol, v. 24, n. 8, p. 1474-8, Aug 2004. ISSN 1524-4636 (Electronic)

1079-5642 (Linking). Disponível em: < http://www.ncbi.nlm.nih.gov/pubmed/15178562 >.

YUAN, C. et al. Cumulative effects of hypertension, dyslipidemia, and chronic kidney disease on carotid atherosclerosis in Chinese patients with type 2 diabetes mellitus. J Diabetes Res, v. 2014, p. 179686, 2014. ISSN 2314-6753 (Electronic). Disponível em: < http://www.ncbi.nlm.nih.gov/pubmed/24860832 >.

LUO, $X$. et al. Differences in left and right carotid intima-media thickness and the associated risk factors. Clin Radiol, v. 66, n. 5, p. 393-8, May 2011. ISSN 1365-229X (Electronic)

0009-9260 (Linking). Disponível em: < http://www.ncbi.nlm.nih.gov/pubmed/21324442 >.

31 LAYTON, K. F. et al. Bovine aortic arch variant in humans: clarification of a common misnomer. AJNR Am J Neuroradiol, v. 27, n. 7, p. 1541-2, Aug 2006. ISSN 0195-6108 (Print)

0195-6108 (Linking). Disponível em: < http://www.ncbi.nlm.nih.gov/pubmed/16908576 >.

MICHAUD, M. et al. Proinflammatory cytokines, aging, and age-related diseases. J Am Med Dir Assoc, v. 14, n. 12, p. 877-82, Dec 2013. ISSN 1538-9375 (Electronic)

1525-8610 (Linking). Disponível em: < http://www.ncbi.nlm.nih.gov/pubmed/23792036 >. 
http://www.ncbi.nlm.nih.gov/entrez/query.fcgi?cmd=Retrieve\&db=PubMed\&dopt=Citation \&list uids $=10911963>$. Immunology and Allergy Clinics of North America, v. 23, n. 1, p. 15-39, 2// 2003. ISSN 08898561. Disponivel em: http://www.sciencedirect.com/science/article/pii/S0889856102000565 >.

AMERICAN DIABETES, A. Diagnosis and classification of diabetes mellitus. Diabetes Care, v. 34 Suppl 1, p. S62-9, Jan 2011. ISSN 1935-5548 (Electronic)

0149-5992 (Linking). Disponível em: < http://www.ncbi.nlm.nih.gov/pubmed/21193628 >.

DHHS, D. O. H. A. H. S. Physical Activity Guidelines Advisory Committee report, 2008. To the Secretary of Health and Human Services. Part A: executive summary. Nutr Rev, v. 67, n. 2, p. 114-20, Feb 2009. ISSN 1753-4887 (Electronic)

0029-6643 (Linking). Disponível em: < http://www.ncbi.nlm.nih.gov/pubmed/19178654 >.

37 BACKINGER, C. L. et al. Use of other tobacco products among U.S. adult cigarette smokers: prevalence, trends and correlates. Addict Behav, v. 33, n. 3, p. 472-89, Mar 2008. ISSN 03064603 (Print)

0306-4603 (Linking). Disponível em: < http://www.ncbi.nlm.nih.gov/pubmed/18053653 >. 
Table 1. Anthropometric, clinical and metabolic variables of the sample.

\begin{tabular}{lc}
\hline Variable & Value \\
\hline n, subjects & 168 \\
Male, $\%$ & 39.9 \\
Age, years & $73.1 \pm 9.0$ \\
BMI, kg.m ${ }^{-2}$ & $27.7 \pm 5.3$ \\
WC, cm & $97.4 \pm 11.5$ \\
Glucose level, mg.dl ${ }^{-1}$ & $103.2 \pm 27.7$ \\
HbAlc, \% & $5.9 \pm 1.0$ \\
Insulin, mUI/mL & $9.3 \pm 10.0$ \\
HOMA index & $2.6 \pm 3.8$ \\
DM2, \% & 22.6 \\
Total cholesterol, mg.dl & $193.4 \pm 39.9$ \\
VLDL-c, mg.dl ${ }^{-1}$ & $29.8 \pm 15.2$ \\
HDL-c, mg. dl ${ }^{-1}$ & $48.1 \pm 10.9$ \\
LDL-c, mg.dl & -1 \\
Triglycerides, mg.dl ${ }^{-1}$ & $115.3 \pm 33.7$ \\
SBP, mm Hg & $141.2 \pm 64.7$ \\
DBP, mm Hg & $134.8 \pm 19.5$ \\
SAH, \% & $80.6 \pm 11.1$ \\
CRP, mg/dL & 77.4 \\
TSH, mIU/L & $3.5 \pm 5.6$ \\
Homocysteine, $\mu$ mol/L & $2.5 \pm 2.2$ \\
Previous stroke, \% & $12.8 \pm 8.8$ \\
Previous AMI, \% & 9.5 \\
Previous CAD, \% & 4.2 \\
Sedentary, \% & 1.8 \\
Smoker, \% & 60.7 \\
Right cIMT, mm & 36.9 \\
Left cIMT, mm & $1.36 \pm 1.63$ \\
& $1.21 \pm 1.13$ \\
\hline
\end{tabular}

Data are expressed as average \pm standard deviation for continuous parameters or relative frequencies for categorical features. $\mathrm{BMI}=$ body mass index; $\mathrm{WC}=$ waist circumference; $\mathrm{HbA} 1 \mathrm{c}=$ glycated hemoglobin A1c; HOMA = Homeostatic model assessment; DM2 = type 2 diabetes mellitus; VLDL$\mathrm{c}=$ very low density lipoprotein cholesterol; LDL-c $=$ low density lipoprotein cholesterol; HDL-c $=$ high density lipoprotein cholesterol, $\mathrm{SBP}=$ systolic blood pressure; $\mathrm{DBP}=$ diastolic blood pressure; $\mathrm{SAH}=$ systemic arterial hypertension; $\mathrm{CRP}=\mathrm{C}$-reactive protein; $\mathrm{TSH}=$ thyroid-stimulating hormone; $\mathrm{AMI}=$ acute myocardial infarction; $\mathrm{CAD}=$ coronary artery disease , cIMT = carotid intima-media thickness. 
Table 2. Correlation analysis of raw levels of serum cytokines and of cIMT scores across categorical clinical/healthcare features of the subjects at admission.

\begin{tabular}{|c|c|c|c|c|c|c|c|c|c|c|}
\hline & \multicolumn{5}{|c|}{ Clinical } & \multicolumn{5}{|c|}{ Healthcare } \\
\hline & Sex & DM2 & SAH & Stroke & AMI & Smoker & Sedentary & $\begin{array}{l}\text { anti SAH } \\
\text { drug }\end{array}$ & $\begin{array}{l}\text { antilipemic } \\
\text { drug }\end{array}$ & $\begin{array}{c}\text { anti DM2 } \\
\text { drug }\end{array}$ \\
\hline $\mathrm{IFN} \gamma, \mathrm{pg} / \mathrm{ml}$ & $.090 ; .266$ & $-.070 ; .382$ & $.107 ; .182$ & $.066 ; .410$ & $-.056 ; .487$ & $.155 ; .055$ & $.076 ; .344$ & $.053 ; .511$ & $.074 ; .361$ & $-.058 ; .476$ \\
\hline IL16, pg/ml & $-.013 ; .869$ & $.017 ; .834$ & $-.077 ; .326$ & $-.091 ; .250$ & $.016 ; .844$ & $.082 ; .304$ & $.025 ; .751$ & $-.089 ; .259$ & $.001 ; .992$ & $.035 ; .654$ \\
\hline IL2, pg/ml & $.061 ; .450$ & $-.054 ; .503$ & $.057 ; .477$ & $.096 ; .231$ & $.056 ; .487$ & $.122 ; .131$ & $.099 ; .217$ & $.032 ; .692$ & $-.000 ; .997$ & $-.097 ; .229$ \\
\hline $\mathrm{IL} 4, \mathrm{pg} / \mathrm{ml}$ & $.022 ; .783$ & $-.031 ; .697$ & $.049 ; .543$ & $.001 ; .991$ & $-.013 ; .868$ & $.102 ; .208$ & $.083 ; .304$ & $.066 ; .413$ & $.068 ; .402$ & $-.051 ; .526$ \\
\hline IL6, pg/ml & $.087 ; .270$ & $-.135 ; .085$ & $-.155 ; .048$ & $-.071 ; .369$ & $.127 ; .107$ & $.002 ; .981$ & $.089 ; .257$ & $-.153 ; .052$ & $.145 ; .064$ & $-.039 ; .620$ \\
\hline IL8, pg/ml & $-.050 ; .523$ & $.032 ; .683$ & $-.025 ; .755$ & $-.057 ; .470$ & $.123 ; .118$ & $-.003 ; .968$ & $.025 ; .750$ & $-.001 ; .991$ & $.002 ; .983$ & $.066 ; .399$ \\
\hline $\mathrm{IL} 10, \mathrm{pg} / \mathrm{ml}$ & $.123 ; .118$ & $.038 ; .630$ & $-.066 ; .401$ & $-.101 ; .200$ & $.002 ; .980$ & $.003 ; .972$ & $.044 ; .575$ & $.004 ; .963$ & $-.126 ; .109$ & $.061 ; .439$ \\
\hline $\mathrm{IL} 12, \mathrm{pg} / \mathrm{ml}$ & $-.033 ; .680$ & $-.023 ; .766$ & $-.069 ; .384$ & $-.162 ; .022$ & $.033 ; .675$ & $-.011 ; .890$ & $-.002 ; .978$ & $-.054 ; .492$ & $-.200 ; .011$ & $.007 ; .926$ \\
\hline IL17a, pg/ml & $-.020 ; .801$ & $.010 ; .905$ & $.118 ; .143$ & $.009 ; .910$ & $-.056 ; .484$ & $.104 ; .198$ & $.128 ; .111$ & $.108 ; .181$ & $.073 ; .362$ & $-.070 ; .383$ \\
\hline TNFŬ pg/ml & $.120 ; .128$ & $-.084 ; .289$ & $-.044 ; .579$ & $-.144 ; .066$ & $.013 ; .869$ & $.110 ; .164$ & $.135 ; .085$ & $.014 ; .854$ & $-.027 ; .728$ & $-.022 ; .776$ \\
\hline Right cIMT, mm & $-.037 ; .708$ & $.091 ; .358$ & $.116 ; .245$ & $.180 ; .068$ & $.089 ; .370$ & $.077 ; .044$ & $.108 ; .277$ & $.220 ; .026$ & $.076 ; .443$ & $.062 ; .537$ \\
\hline Left cIMT, mm & $-.010 ; .922$ & $-.034 ; .745$ & $-.049 ; .637$ & $.246 ; .016$ & $.098 ; .341$ & $.090 ; .389$ & $.245 ; .016$ & $-.050 ; .629$ & $.037 ; .724$ & $-.060 ; .559$ \\
\hline
\end{tabular}

The Spearmanôs correlation test was used. Data are expressed in correlation index and significance level $(r ; P)$. DM2 = type 2 diabetes mellitus; SAH $=$ systemic arterial hypertension; AMI = acute myocardial infarction; IFN = interferon; IL = interleukin; TNF = tumor necrosis factor; cIMT = carotid intimamedia thickness. 

Table 3. Correlation analysis of logarithmically-transformed levels of serum cytokines and of raw cIMT scores across continuous clinical/biochemical features of the 168 subjects at admission.

\begin{tabular}{|c|c|c|c|c|c|c|c|c|c|c|}
\hline & \multicolumn{4}{|c|}{ Clinical } & \multicolumn{6}{|c|}{ Biochemical } \\
\hline & Age & WC & SBP & DBP & TC & VLDL-c & HDL-c & TGL & HOMA & HbA1c \\
\hline $\log _{10} \mathrm{IFN} \gamma$ & $.046 ; .568$ & $-.011 ; .894$ & $.098 ; .223$ & $.087 ; .282$ & $-.022 ; .785$ & $.063 ; .438$ & $-.115 ; .153$ & $.086 ; .288$ & $.058 ; .477$ & $-.030 ; .716$ \\
\hline $\log _{10} \mathrm{IL} 16$ & $.091 ; .251$ & $.012 ; .880$ & $.078 ; .325$ & $.037 ; .641$ & $.154 ; .050$ & $.076 ; .339$ & $.247 ; .001$ & $.094 ; .232$ & $.029 ; .717$ & $.015 ; .851$ \\
\hline $\log _{10} \mathrm{IL} 2$ & $-.044 ; .587$ & $.040 ; .623$ & $.009 ; .914$ & $.067 ; .404$ & $-.006 ; .942$ & $.006 ; .945$ & $.010 ; .902$ & $.063 ; .432$ & $-.006 ; .939$ & $-.110 ; .177$ \\
\hline $\log _{10} \mathrm{IL} 4$ & $-.069 ; .392$ & $.061 ; .456$ & $.119 ; .138$ & $.155 ; .053$ & $-.002 ; .984$ & $.102 ; .207$ & $-.037 ; .645$ & $.122 ; .128$ & $.025 ; .758$ & $-.049 ; .151$ \\
\hline $\log _{10} \mathrm{IL} 6$ & $.073 ; .353$ & $-.089 ; .262$ & $-.104 ; .185$ & $-.074 ; .348$ & $.069 ; .384$ & $-.136 ; .084$ & $.218 ; .004$ & $-.132 ; .094$ & $-.177 ; .025$ & $-.167 ; .036$ \\
\hline $\log _{10} \mathrm{IL} 8$ & $.138 ; .078$ & $-.002 ; .983$ & $.034 ; .669$ & $-.032 ; .688$ & $.062 ; .435$ & $-.002 ; .979$ & $.110 ; .164$ & $-.022 ; .779$ & $.017 ; .830$ & $-.014 ; .860$ \\
\hline $\log _{10} \mathrm{IL} 10$ & $-.020 ; .800$ & $.014 ; .862$ & $.024 ; .765$ & $.043 ; .587$ & $.182 ; .020$ & $.089 ; .258$ & $.127 ; .108$ & $.140 ; .075$ & $.064 ; .426$ & $.085 ; .290$ \\
\hline $\log _{10} \mathrm{IL} 12$ & $-.111 ; .080$ & $.024 ; .763$ & $.070 ; .378$ & $.062 ; .429$ & $.150 ; .055$ & $.092 ; .246$ & $.087 ; .270$ & $.095 ; .226$ & $.001 ; .993$ & $.072 ; .369$ \\
\hline $\log _{10} \mathrm{IL} 17 \mathrm{a}$ & $.056 ; .491$ & $-.062 ; .443$ & $.115 ; .154$ & $.132 ; .104$ & $-.059 ; .466$ & $.055 ; .501$ & $-.042 ; .606$ & $.087 ; .279$ & $.006 ; .940$ & $.006 ; .939$ \\
\hline $\log _{10}$ TNFŬ & $.077 ; .328$ & $.044 ; .583$ & $.032 ; .689$ & $.015 ; .846$ & $.108 ; .177$ & $.048 ; .544$ & $.179 ; .022$ & $.071 ; .365$ & $-.063 ; .430$ & $-.068 ; .399$ \\
\hline Right cIMT, mm & $.196 ; .048$ & $-.105 ; .293$ & $.014 ; .886$ & $.028 ; .781$ & $-.094 ; .344$ & $-.084 ; .400$ & $.094 ; .346$ & $-.067 ; .502$ & $-.013 ; .902$ & $-.061 ; .548$ \\
\hline Left cIMT, mm & $.229 ; .025$ & $-.094 ; .365$ & $.032 ; .757$ & $.066 ; .521$ & $.054 ; .600$ & $-.024 ; .813$ & $.116 ; .262$ & $-.011 ; .917$ & $.078 ; .459$ & $-.049 ; .641$ \\
\hline
\end{tabular}

The Pearsonô correlation test was used. Data are expressed in correlation index and significance level $(r ; P)$. WC = waist circumference; $\mathrm{SBP}=$ systolic blood pressure; DBP = diastolic blood pressure; TC = total cholesterol; VLDL-c = very low density lipoprotein cholesterol; HDL-c $=$ high density lipoprotein cholesterol; HOMA = Homeostatic model assessment; HbA1c = glycated hemoglobin A1c; Log = logarithmically-transformed data; IFN = interferon; IL = interleukin; $\mathrm{TNF}=$ tumor necrosis factor; cIMT = carotid intima-media thickness . 

Table 4. Correlation analyses between logarithmically-transformed levels of serum cytokines and raw cIMT scores of the 168 subjects at admission.

\begin{tabular}{|c|c|c|}
\hline & \multicolumn{2}{|c|}{ cIMT, mm } \\
\hline & Left & Right \\
\hline $\log _{10} \mathrm{IFN} \gamma, \mathrm{pg} / \mathrm{ml}$ & $.113 ; .292$ & $.175 ; .088$ \\
\hline $\log _{10} \mathrm{IL} 16, \mathrm{pg} / \mathrm{ml}$ & $.443 ;<.001$ & $.185 ; .065$ \\
\hline $\log _{10} \mathrm{IL} 2, \mathrm{pg} / \mathrm{ml}$ & $.188 ; .078$ & $.155 ; .132$ \\
\hline $\log _{10} \mathrm{IL} 4, \mathrm{pg} / \mathrm{ml}$ & $.146 ; .172$ & $.085 ; .408$ \\
\hline $\log _{10} \mathrm{IL} 6, \mathrm{pg} / \mathrm{ml}$ & $.330 ; .001$ & $.062 ; .541$ \\
\hline $\log _{10} \operatorname{IL} 8, \mathrm{pg} / \mathrm{ml}$ & $.291 ; .005$ & $.171 ; .089$ \\
\hline $\log _{10} \mathrm{IL} 10, \mathrm{pg} / \mathrm{ml}$ & $.348 ; .001$ & $.135 ; .181$ \\
\hline $\log _{10} \mathrm{IL} 12, \mathrm{pg} / \mathrm{ml}$ & $.132 ; .208$ & $-.177 ; \quad .079$ \\
\hline $\log _{10} \operatorname{IL} 17 \mathrm{a}, \mathrm{pg} / \mathrm{ml}$ & $.124 ; .247$ & $-.240 ; \quad .019$ \\
\hline $\log _{10} \mathrm{TNFŬ}, \mathrm{pg} / \mathrm{ml}$ & $.505 ;<.001$ & $-.248 ; \quad .013$ \\
\hline
\end{tabular}

The Pearsonô correlation test was used. Data are expressed in correlation index and significance level $(r ; P) . \log =$ logarithmically-transformed data; IFN = interferon; $\mathrm{IL}=$ interleukin; $\mathrm{TNF}=$ tumor necrosis factor; cIMT $=$ carotid intima-media thickness. 

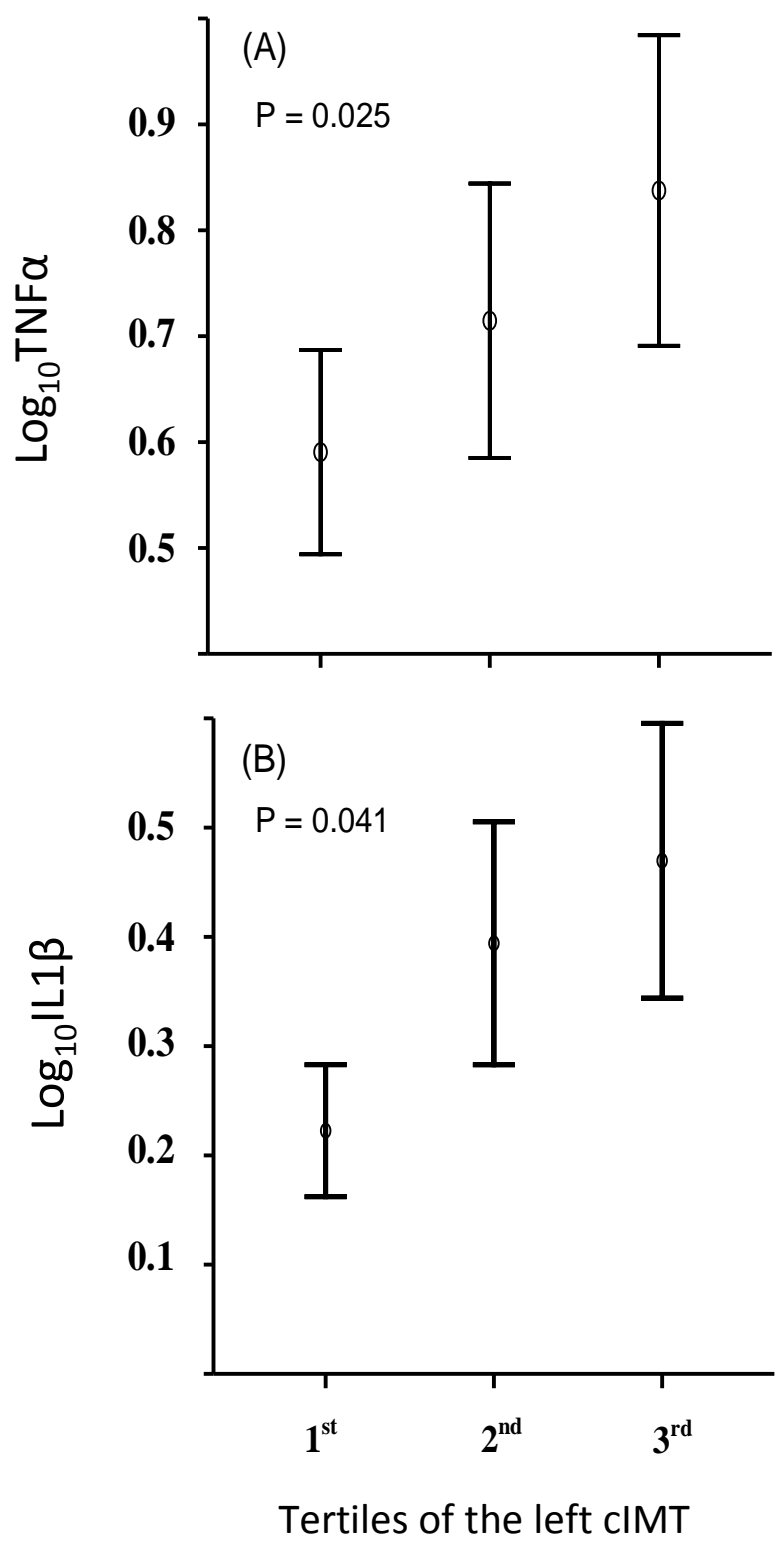

Figure 1. Comparison of log-transformed circulating levels of TNFǓ(A) and IL1 6 (B) across the individuals grouped in increasing tertiles of the left carotid intima-media thickness (cIMT). Significance was verified by ANOVA with adjustment to HDL-c. Vertical bars represent intervals of one standard error. 
